\title{
Unsteady motion of a long bubble or droplet in a self-rewetting system
}

\author{
B. R. Duffy, ${ }^{1, *}$ S. K. Wilson, ${ }^{1, \dagger}$ J. J. A. Conn,${ }^{1}$ and K. Sefiane $e^{2, \ddagger}$ \\ ${ }^{1}$ Department of Mathematics and Statistics, University of Strathclyde, \\ Livingstone Tower, 26 Richmond Street, Glasgow G1 1XH, UK \\ ${ }^{2}$ School of Engineering, University of Edinburgh, \\ The King's Buildings, Mayfield Road, Edinburgh EH9 3FB, UK
}

(Dated: 16th August 2018, revised 26th October 2018)

\begin{abstract}
Motivated by the potential use of self-rewetting fluids (i.e. fluids that exhibit a non-monotonic variation of surface tension with temperature) in various heat-transfer applications, in the present work we formulate and analyse a theoretical model for the unsteady motion of a long bubble or droplet in a self-rewetting system in a non-uniformly heated tube due to a combination of Marangoni effects due to the variation of surface tension with temperature, gravitational effects due to the density difference between the two fluids, and an imposed background flow along the tube. We find that the evolution of the shape (but not of the position) of the bubble or droplet is driven entirely by Marangoni effects, and depends on the initial value of its radius in relation to a critical value. In the case in which Marangoni effects are absent the bubble or droplet always moves with constant velocity without changing shape. In the case in which only Marangoni effects are present the bubble or droplet either always moves away from or always moves towards the position of minimum surface tension; in the latter case it ultimately fills the entire cross-section of the tube at a final stationary position which is closer to the position of minimum surface tension than its original position. In the cases in which either only Marangoni effects and gravitational effects or only Marangoni effects and background-flow effects are present the competition between the two effects can lead to a nonmonotonic evolution of the position of the centre of mass of the bubble or droplet. The behaviour of a self-rewetting system described in the present work is qualitatively different from that for ordinary fluids, in which case the bubble or droplet always moves with constant velocity without changing shape.
\end{abstract}

\footnotetext{
* b.r.duffy@strath.ac.uk

$\dagger$ Author for correspondence; s.k.wilson@strath.ac.uk

‡ k.sefiane@ed.ac.uk
} 


\section{INTRODUCTION}

The thermocapillary-driven (i.e. surface-tension-gradient-driven, hereafter referred to simply as "Marangonidriven") motion of bubbles and droplets plays an important role in a wide variety of practical situations including, for example, heat-transfer and material-processing applications. For most "ordinary" fluids, surface tension, here denoted by $\sigma$, is a monotonically (typically, to a good approximation, linearly) decreasing function of temperature, here denoted by $T$ (see, for example, Figure 1(a)), and as a result Marangoni effects draw fluid towards regions with lower temperature. However, for many years it has been known (see, for example, the pioneering work of Vochten and Petre [1], Petre and Azouni [2], Legros [3], and Vázquez, Alvarez and Navaza [4]) that this behaviour is not universal, and that there are certain fluids, notably dilute aqueous solutions of certain long-chain alcohols, that exhibit a non-monotonic (typically, to a good approximation, locally quadratic) variation of surface tension with temperature, with a minimum value, here denoted by $\sigma_{\min }$, at a well-defined value of temperature, here denoted by $T_{\min }$ (see, for example, Figure 1(b)). In particular, for temperatures above $T_{\min }$ the surface tension of such fluids is an increasing function of temperature, and as a result Marangoni effects draw fluid towards regions with higher temperature. This unusual behaviour has stimulated interest in the potential use of such fluids in various heat-transfer applications, such as pool boiling, spray boiling and heat pipes, because in these contexts it tends to reduce dry-out, and hence to enhance the overall heat transfer, by rewetting hot surfaces, and so has led to them being termed "self-rewetting"

fluids (see, for example, the work of Zhang [5], Abe, Iwasaki and Tanaka [6, 7], Abe [8], Savino and collaborators [9-15], Hu et al. [16], Hu, Zhang and Wang [17], and Wu [18]).

There have been a few studies of the effect of a non-monotonic variation of surface tension with temperature on the dynamics of fluid films (notably the early work by Oron and Rosenau [19], Slavtchev and Miladinova [20], and the more recent work by Batson, Agnon and Oron [21]). However, in contrast to the large body of work on the motion of both confined and unconfined bubbles and droplets in or of an ordinary fluid in the presence of significant Marangoni effects (see, for example, the work of Young, Goldstein and Block [22], Balasubramaniam and Chai [23], Ehrhard and Davis [24], Wilson [25, 26], Smith [27], Balasubramaniam and Subramanian [28], Mazouchi and Homsy [29, 30], Lajeunesse and Homsy [31], Dunn et al. [32], Katz et al. [33], and Karapetsas, Sahu and Matar [34]), until recently there has been virtually no work on the motion of a bubble or droplet in or of a self-rewetting fluid. These studies show that the behaviour of a bubble or droplet in or of a self-rewetting fluid can be qualitatively different from that for an ordinary fluid. Specifically, the theoretical studies by Karapetsas et al. [35] of the motion of a droplet on a heated substrate, and by Tripathi et al. [36] of the rise of a bubble in a vertical channel, as well as the experimental studies by Shanahan and Sefiane [37] of the motion of a bubble in a mean flow, by Mamalis, Koutsos and Sefiane [38] of the motion of a droplet on a heated inclined substrate, by Mamalis, Koutsos and Sefiane [39] of the rise of a bubble in a vertical micro-channel, and by Mamalis, Koutsos and Sefiane [40] of the spreading of a droplet on a heated substrate, all demonstrate this. In the present work we seek to bring further insight into this problem by formulating and analysing a theoretical model for the unsteady motion of a long bubble or droplet in a self-rewetting system in a non-uniformly heated tube. In particular, we find that the evolution of the shape (but not of the position) of the bubble or droplet is driven entirely by Marangoni effects, and depends on the initial value of its radius in relation to a critical value. 

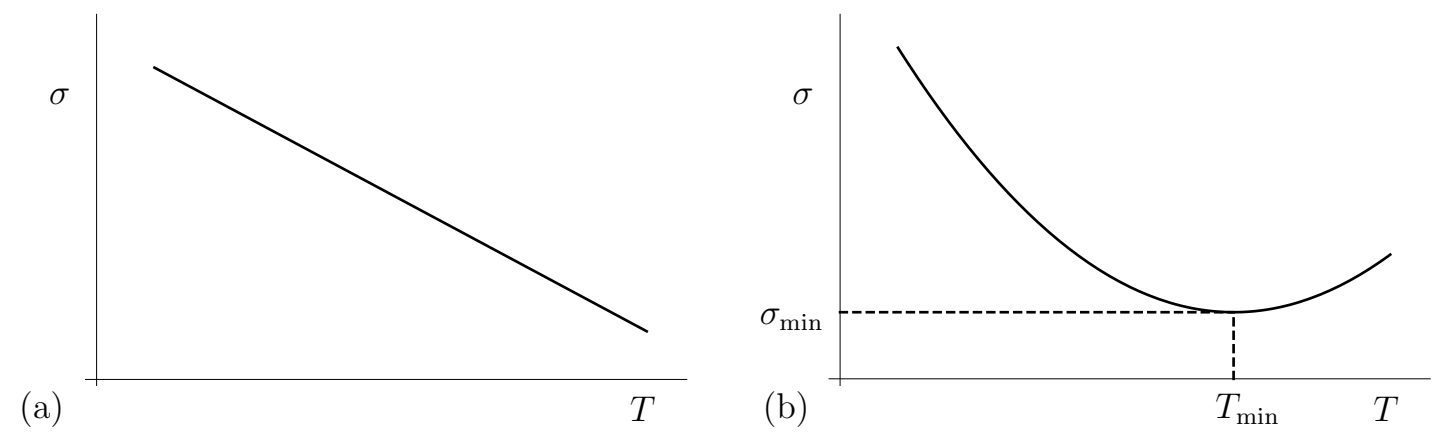

FIG. 1. Sketches of surface tension $\sigma$ as a function of temperature $T$ for (a) an ordinary and (b) a self-rewetting fluid.

\section{PROBLEM FORMULATION}

Consider the unsteady motion of a long axisymmetric bubble or droplet of fluid (denoted fluid 1), with constant volume $V$, uniform radius $b(t)$, and length $L(t)$, where $t$ denotes time, in a second fluid (denoted fluid 2), all contained within a non-uniformly heated vertical tube of constant radius $a$, where $0<b(t)<a \ll L(t)$. The motion is due to a combination of Marangoni effects due to the variation of surface tension with temperature, gravitational effects due to the density difference between the two fluids, and an imposed background flow along the tube with prescribed axial volume flux $Q_{\mathrm{b}}$. For brevity, henceforth we will use the general term "droplet", rather than a "bubble or droplet", except when explicitly referring to the special case in which fluid 1 is inviscid.

Figure 2 shows a sketch of the geometry of the problem. We choose a cylindrical polar coordinate system, with the $z$-axis vertically upwards along the centre-line of the tube, and with the origin $z=0$ at the vertical position at which the wall temperature takes the value $T_{\min }$. With respect to this coordinate system, the velocity, pressure, and temperature of fluid $i$ for $i=1,2$ are denoted by $\mathbf{u}_{i}=\left(u_{i}, w_{i}\right), p_{i}(r, z, t)$ and $T_{i}(r, z, t)$, respectively, where $u_{i}(r, z, t)$ and $w_{i}(r, z, t)$ denote the radial and the axial velocity, respectively. The positions of the front and back of the droplet are denoted by $z=z_{\mathrm{f}}(t)$ and $z=z_{\mathrm{b}}(t)=z_{\mathrm{f}}(t)-L(t)\left(<z_{\mathrm{f}}(t)\right)$, respectively, in terms of which the position of the centre of mass of the droplet is given by $z=c(t)=\left(z_{\mathrm{f}}(t)+z_{\mathrm{b}}(t)\right) / 2$.

We assume that both fluids are incompressible, with constant bulk material properties which may differ between the two fluids. Specifically, fluid $i$ for $i=1,2$ has constant density $\rho_{i}$, dynamic viscosity $\mu_{i}$, heat capacity $c_{\mathrm{p}, i}$, and thermal conductivity $k_{i}$.

To model the self-rewetting properties of the system we take the surface tension of the interface between the two fluids, $\sigma$, to be a quadratic function of temperature $T$ with a minimum value $\sigma=\sigma_{\min }$ at $T=T_{\min }$ given by

$$
\sigma=\sigma_{\min }+\frac{\gamma}{2}\left(T-T_{\min }\right)^{2},
$$

where $\gamma=\mathrm{d}^{2} \sigma / \mathrm{d} T^{2}>0$ is a positive constant.

A linear temperature distribution is imposed on the tube wall, and so

$$
T_{2}=T_{\min }+\beta z \quad \text { on } \quad r=a,
$$

where the constant $\beta$ is the imposed temperature gradient, which may be positive or negative depending on whether the top or the bottom of the tube is hotter. However, since $\sigma$ is a quadratic function of $T-T_{\min }$, we will find that 


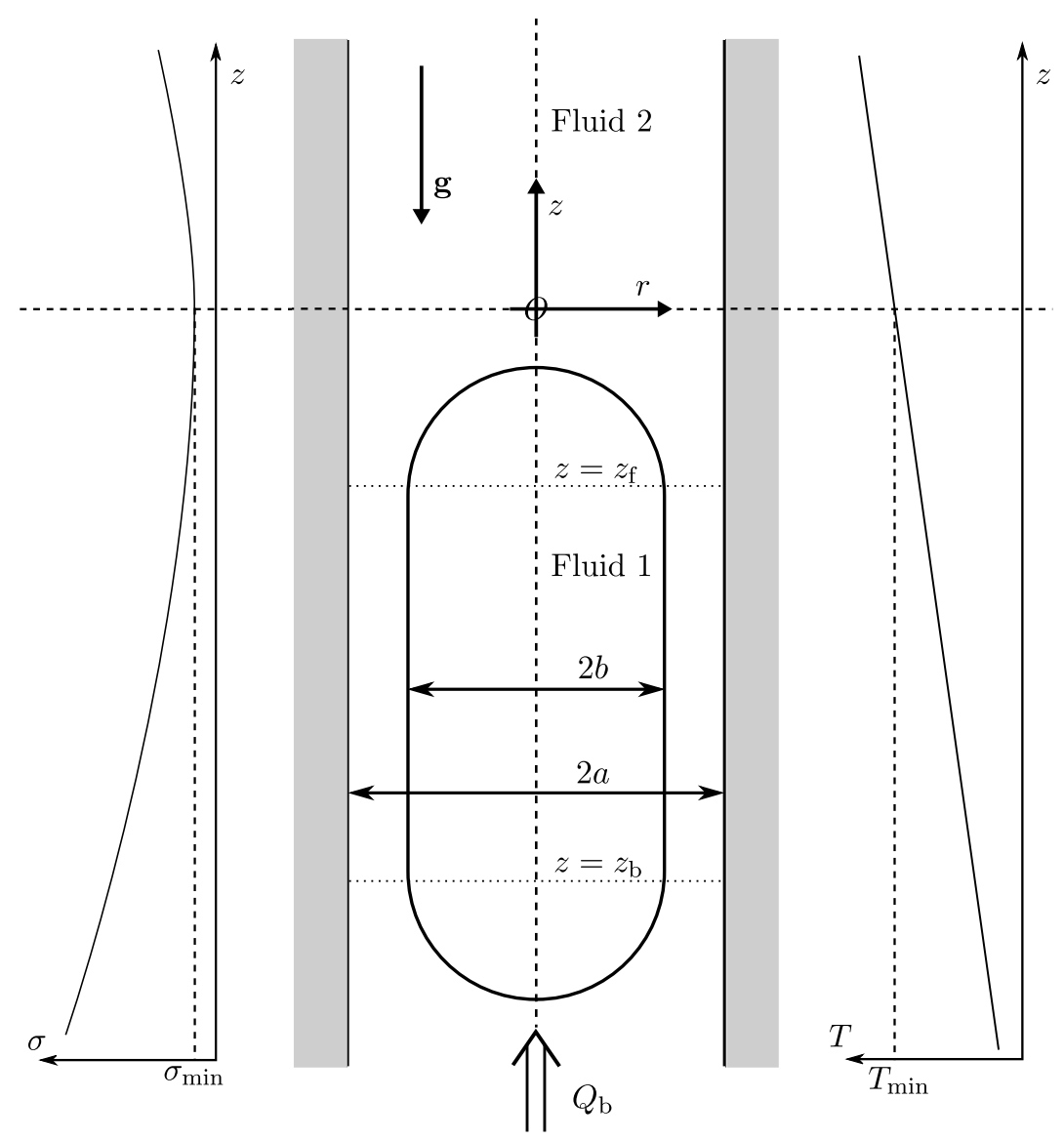

FIG. 2. Sketch of the geometry of the problem: a long axisymmetric bubble or droplet of fluid (fluid 1) of uniform radius $b(t)$ moving in a second fluid (fluid 2), all contained within a non-uniformly heated vertical tube of constant radius $a$. The linear temperature distribution $T$ imposed on the tube wall and the resulting quadratic variation of surface tension $\sigma$ are shown the right and the left of the figure, respectively.

it depends on $\beta$ like $\beta^{2}$ (rather than like $\beta$, as it would do for ordinary fluids for which $\sigma$ is a linearly decreasing function of $T$ ), and therefore we may, without loss of generality, take $\beta$ to be positive (i.e. we may, without loss of generality, consider the situation shown in Figure 2 in which the top of the tube is hotter than the bottom).

Since the droplet is long compared to the radius of the tube, its aspect ratio $\varepsilon$, defined by

$$
\varepsilon=\frac{a}{\ell} \ll 1
$$

is small, where

$$
\ell=\frac{V}{\pi a^{2}}
$$

is a characteristic axial length scale of the problem, chosen such that the scaled volume of the droplet is unity. It is then appropriate to nondimensionalise and scale all relevant quantities in the system according to

$$
\left.\begin{array}{c}
r^{*}=\frac{r}{a}, \quad b^{*}=\frac{b}{a}, \quad z^{*}=\frac{\varepsilon z}{a}, \quad L^{*}=\frac{\varepsilon L}{a}, \quad t^{*}=\frac{\varepsilon W t}{a}, \quad u_{i}^{*}=\frac{u_{i}}{\varepsilon W}, \quad w_{i}^{*}=\frac{w_{i}}{W}, \quad p_{i}^{*}=\frac{\varepsilon a p_{i}}{\mu_{2} W}, \\
Q_{i}^{*}=\frac{Q_{i}}{\pi a^{2} W}, \quad Q_{\mathrm{b}}^{*}=\frac{Q_{\mathrm{b}}}{\pi a^{2} W}, \quad T_{i}^{*}=\frac{T_{i}-T_{\min }}{T_{\min }}, \quad \beta^{*}=\frac{a \beta}{\varepsilon T_{\min }}, \quad \sigma^{*}=\frac{\sigma}{\sigma_{\min }},
\end{array}\right\}
$$


where the stars denote dimensionless quantities, $Q_{i}^{*}$ for $i=1,2$ denote the axial volume fluxes of fluid 1 and fluid 2 , respectively, given by

$$
Q_{1}^{*}=2 \int_{0}^{b^{*}} r^{*} w_{1}^{*} \mathrm{~d} r^{*}, \quad Q_{2}^{*}=2 \int_{b^{*}}^{1} r^{*} w_{2}^{*} \mathrm{~d} r^{*},
$$

and $W$ is the characteristic axial velocity scale. The particular form for $W$ may be chosen in several different ways, such as $W=\varepsilon \gamma T_{\min }^{2} / \mu_{2}$ when Marangoni effects are important, $W=\left(\rho_{2}-\rho_{1}\right) g a^{2} / \mu_{2}$ when gravitational effects are important, or $W=Q_{\mathrm{b}} / \pi a^{2}$ when the background-flow effects are important, and so we leave $W$ unspecified here.

Away from the ends of the droplet and at leading order in the limit $\varepsilon \rightarrow 0$, the velocity, pressure and temperature of the two fluids satisfy

$$
\begin{aligned}
\frac{1}{r^{*}} \frac{\partial}{\partial r^{*}}\left(r^{*} u_{1}^{*}\right)+\frac{\partial w_{1}^{*}}{\partial z^{*}}=0, & \frac{1}{r^{*}} \frac{\partial}{\partial r^{*}}\left(r^{*} u_{2}^{*}\right)+\frac{\partial w_{2}^{*}}{\partial z^{*}}=0, \\
\frac{\partial p_{1}^{*}}{\partial r^{*}}=0, & \frac{\partial p_{2}^{*}}{\partial r^{*}}=0, \\
\frac{m}{r^{*}} \frac{\partial}{\partial r^{*}}\left(r^{*} \frac{\partial w_{1}^{*}}{\partial r^{*}}\right)=\frac{\partial p_{1}^{*}}{\partial z^{*}}+\rho_{1}^{*}, & \frac{1}{r^{*}} \frac{\partial}{\partial r^{*}}\left(r^{*} \frac{\partial w_{2}^{*}}{\partial r^{*}}\right)=\frac{\partial p_{2}^{*}}{\partial z^{*}}+\rho_{2}^{*}, \\
\frac{1}{r^{*}} \frac{\partial}{\partial r^{*}}\left(r^{*} \frac{\partial T_{1}^{*}}{\partial r^{*}}\right)=0, & \frac{1}{r^{*}} \frac{\partial}{\partial r^{*}}\left(r^{*} \frac{\partial T_{2}^{*}}{\partial r^{*}}\right)=0,
\end{aligned}
$$

representing balances of mass, linear momentum in the radial direction, linear momentum in the axial direction, and energy, respectively, and where we have assumed that the reduced Reynolds numbers $\varepsilon^{2} \operatorname{Re}_{i}$ and the reduced Péclet numbers $\varepsilon^{2} \mathrm{Pe}_{i}$ for $i=1,2$, defined by

$$
\varepsilon^{2} \operatorname{Re}_{i}=\frac{\varepsilon \rho_{i} a W}{\mu_{i}} \ll 1, \quad \varepsilon^{2} \mathrm{Pe}_{i}=\frac{\varepsilon \rho_{i} c_{\mathrm{p}, i} a W}{k_{i}} \ll 1,
$$

in both fluids are small, and have defined the viscosity ratio,

$$
m=\frac{\mu_{1}}{\mu_{2}}
$$

along with the scaled densities of fluid 1 and fluid 2, respectively,

$$
\rho_{1}^{*}=\frac{\rho_{1} g a^{2}}{\mu_{2} W}, \quad \rho_{2}^{*}=\frac{\rho_{2} g a^{2}}{\mu_{2} W},
$$

and the scaled density difference between the two fluids,

$$
\Delta \rho^{*}=\frac{\left(\rho_{2}-\rho_{1}\right) g a^{2}}{\mu_{2} W} .
$$

The special cases of an inviscid bubble and of an inviscid surrounding fluid correspond to $m=0$ and the limit $m \rightarrow \infty$, respectively.

Equations (7)-(10) are subject to the following leading-order boundary conditions. At the axis of symmetry, $r^{*}=0$,

$$
\begin{gathered}
u_{1}^{*}=0, \\
\frac{\partial w_{1}^{*}}{\partial r^{*}}=0, \\
\frac{\partial T_{1}^{*}}{\partial r^{*}}=0 .
\end{gathered}
$$


At the tube wall, $r^{*}=1$,

$$
\begin{gathered}
u_{2}^{*}=0, \\
w_{2}^{*}=0, \\
T_{2}^{*}=\beta^{*} z^{*},
\end{gathered}
$$

which are a no-penetration condition, a no-slip condition, and the imposed wall temperature, respectively. At the interface between the two fluids, $r^{*}=b^{*}$,

$$
\begin{gathered}
u_{1}^{*}=u_{2}^{*}, \\
w_{1}^{*}=w_{2}^{*}, \\
T_{1}^{*}=T_{2}^{*}, \\
\kappa \frac{\partial T_{1}^{*}}{\partial r^{*}}=\frac{\partial T_{2}^{*}}{\partial r^{*}}, \\
p_{1}^{*}-p_{2}^{*}=\frac{1}{b^{*}}\left(\frac{1}{C}+\frac{M}{2 \beta^{2}} T_{1}^{* 2}\right), \\
\frac{\partial w_{2}^{*}}{\partial r^{*}}-m \frac{\partial w_{1}^{*}}{\partial r^{*}}=-\frac{M}{\beta^{2}} T_{1}^{*} \frac{\partial T_{1}^{*}}{\partial z^{*}},
\end{gathered}
$$

which are continuity of radial velocity, axial velocity, temperature and heat flux, and balances of normal stresses and tangential stresses, respectively, where we have defined the thermal conductivity ratio,

$$
\kappa=\frac{k_{1}}{k_{2}},
$$

an appropriate capillary number,

$$
C=\frac{\mu_{2} W}{\varepsilon \sigma_{\min }}
$$

and an appropriate Marangoni number,

$$
M=\frac{\varepsilon \gamma \beta^{2} T_{\min }^{2}}{\mu_{2} W}
$$

Global conservation of mass requires that the total flux of the two fluids must be equal to the prescribed flux of the imposed background flow, $Q_{\mathrm{b}}^{*}$, i.e.

$$
Q_{1}^{*}+Q_{2}^{*}=Q_{\mathrm{b}}^{*}
$$

In particular, in the special case of a closed tube with no background flow, $Q_{\mathrm{b}}^{*}=0$, and so $Q_{1}^{*}=-Q_{2}^{*}$.

Note that, whereas $\Delta \rho^{*}$ and $Q_{\mathrm{b}}^{*}$ may be either positive, negative or zero, $M$ must, by definition, always be nonnegative.

The radius of the droplet, $b^{*}$, satisfies the kinematic condition

$$
2 b^{*} \frac{\mathrm{d} b^{*}}{\mathrm{~d} t^{*}}+\frac{\partial Q_{1}^{*}}{\partial z^{*}}=0, \quad \text { or, equivalently, } \quad 2 b^{*} \frac{\mathrm{d} b^{*}}{\mathrm{~d} t^{*}}-\frac{\partial Q_{2}^{*}}{\partial z^{*}}=0 .
$$


Neglecting the small contributions from its ends, the volume of the droplet is given by $b^{* 2} L^{*}=1$, and hence its length is simply given by

$$
L^{*}=\frac{1}{b^{* 2}}
$$

Note that, since the (dimensional) radius of the droplet must be less than that of the tube (i.e. $0<b<a$ and hence $0<b^{*}<1$ ), its (dimensional) length must be greater than the characteristic axial length scale $\ell$ given by (4) (i.e. $L>\ell$ and hence $\left.L^{*}>1\right)$. Henceforth, for clarity, we drop the stars on dimensionless quantities.

\section{PROBLEM SOLUTION}

The thermal problem, given by (10), (17), (20), (23) and (24), decouples from the hydrodynamic problem and may be solved separately to yield simply

$$
T_{1}=T_{2}=\beta z,
$$

showing that the temperatures of both fluids are radially uniform and identically equal to the imposed wall temperature. In particular, this means that the surface tension given by (1) is a quadratic function of $z$ given by

$$
\sigma=1+\frac{M C}{2} z^{2}
$$

The hydrodynamic problem, given by (7), (8), (9), (15), (16), (18), (19), (21), (22), (25) and (26), may now be solved, leading ultimately to the evolution equations for the shape of the droplet and for its position within the tube (i.e. evolution equations for $b(t), L(t), z_{\mathrm{f}}(t), z_{\mathrm{b}}(t)$ and $\left.c(t)\right)$.

Equation (8) implies that the pressures of both fluids are radially uniform, i.e.

$$
p_{i}=p_{i}(z, t) \quad \text { for } \quad i=1,2 \text {. }
$$

In particular, using (33) and (35), equation (25) shows that

$$
p_{1}-p_{2}=\frac{1}{b}\left(\frac{1}{C}+\frac{M}{2} z^{2}\right) .
$$

Using (35), equation (9) may be integrated to yield expressions for the axial velocities $w_{i}$, which may then be used to determine the radial velocities $u_{i}$ and fluxes $Q_{i}$ of both fluids. The expressions for the pressure gradients and the velocities of both fluids are rather cumbersome, and therefore have been relegated to Appendix A. However, the fluxes take the forms

$$
Q_{1}=M z f_{\mathrm{M}}(b, m)+\Delta \rho f_{\mathrm{G}}(b, m)+Q_{\mathrm{b}} f_{\mathrm{B}}(b, m), \quad Q_{2}=Q_{\mathrm{b}}-Q_{1},
$$

where the functions $f_{j}$ for $j=\mathrm{M}, \mathrm{G}, \mathrm{B}$, which arise in the Marangoni (M), gravitational (G), and background-flow (B) contributions to the fluxes, are given by

$$
f_{\mathrm{M}}(b, m)=\frac{b^{3}}{8}\left[\frac{\left(1-b^{2}\right)\left[(4 m-1) b^{2}-4 m-1\right]}{m-(m-1) b^{4}}-4 \ln b\right],
$$




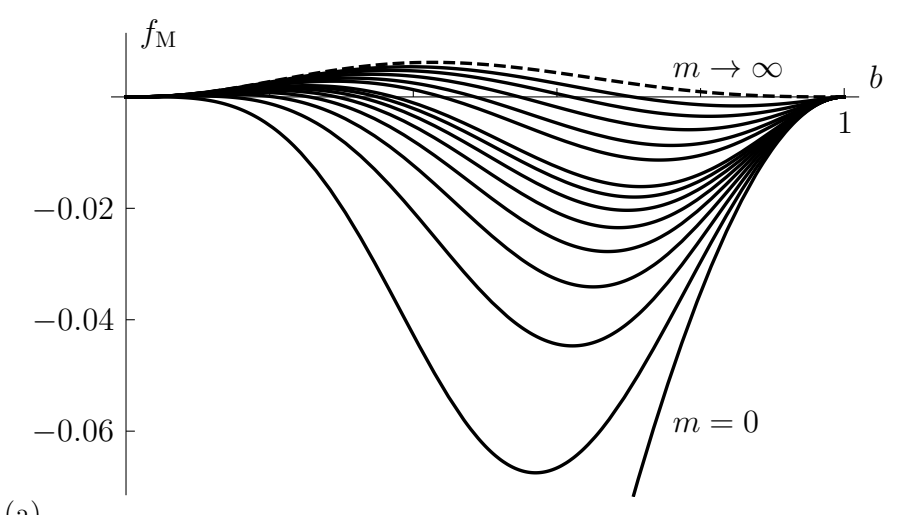

(a)

(b)

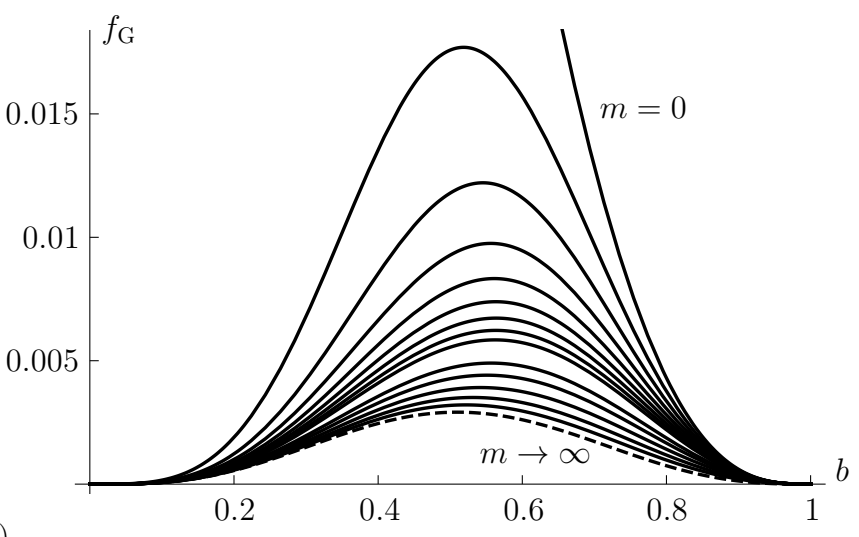

(c)

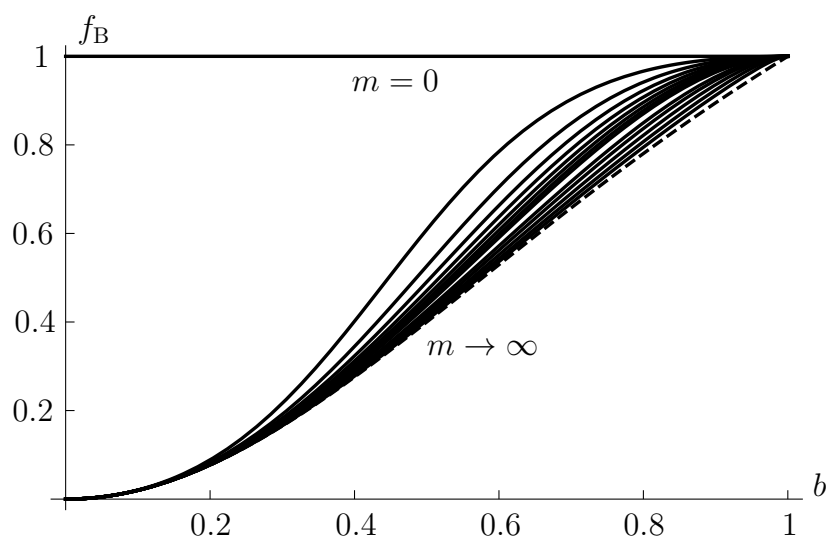

FIG. 3. Plots of the functions $f_{j}$ for $j=\mathrm{M}, \mathrm{G}, \mathrm{B}$ defined by (38)-(40) which arise in the Marangoni (M), gravitational (G), and background-flow (B) contributions to the fluxes appearing in (37) as functions of the droplet radius $b$ for $m=0,1 / 8,1 / 4$, $\ldots, 1,3 / 2,2,5,10$ and in the limit $m \rightarrow \infty$ given by (44)-(46) (the latter shown dashed).

$$
\begin{gathered}
f_{\mathrm{G}}(b, m)=\frac{b^{4}}{8}\left[\frac{\left(1-b^{2}\right)\left[(4 m-3) b^{2}-4 m+1\right]}{m-(m-1) b^{4}}-4 \ln b\right] \quad(>0), \\
f_{\mathrm{B}}(b, m)=\frac{b^{2}\left[2 m-(2 m-1) b^{2}\right]}{m-(m-1) b^{4}} \quad(>0),
\end{gathered}
$$

respectively.

Figure 3 shows $f_{j}$ for $j=\mathrm{M}, \mathrm{G}, \mathrm{B}$ plotted as functions of $b$ for the full range of values of $m$. Each of the $f_{j}$ varies 


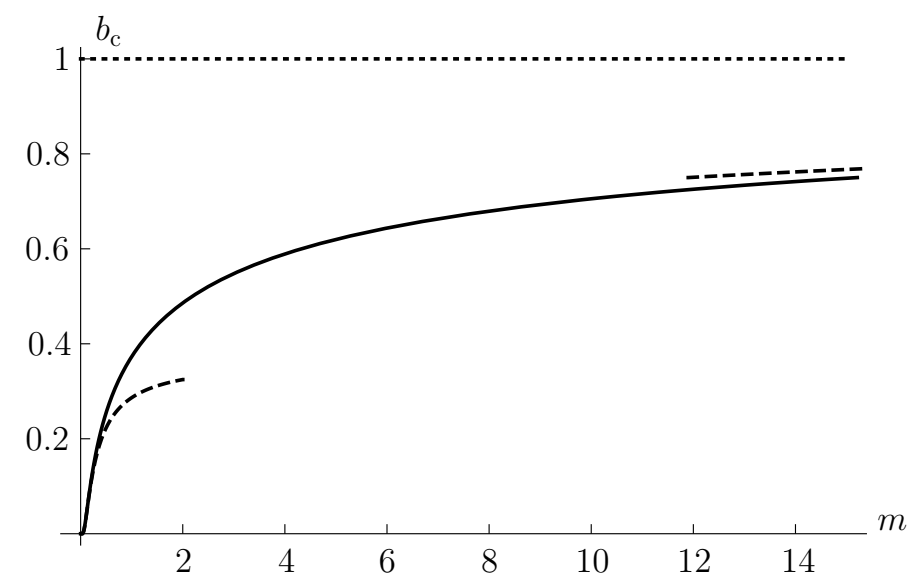

FIG. 4. Plot of the critical droplet radius $b_{\mathrm{c}}$ calculated from (47) as a function of the viscosity ratio $m$. The asymptotic expressions $b_{\mathrm{c}} \sim \exp [-1 /(4 m)-1] \rightarrow 0$ in the limit $m \rightarrow 0^{+}$and $b_{\mathrm{c}} \sim 1-(3 / 2 m)^{1 / 2}+(5 / 4 m) \rightarrow 1^{-}$in the limit $m \rightarrow \infty$ are shown with dashed lines.

monotonically with $m$, with $f_{\mathrm{M}}$ increasing but $f_{\mathrm{G}}$ and $f_{\mathrm{B}}$ decreasing as $m$ increases. In particular, in the special case of an inviscid bubble $(m=0)$ equations $(38)-(40)$ reduce to

$$
\begin{gathered}
f_{\mathrm{M}}(b, 0)=-\frac{1-b^{4}}{8 b}-\frac{b^{3}}{2} \ln b \quad(<0), \\
f_{\mathrm{G}}(b, 0)=\frac{\left(1-b^{2}\right)\left(1-3 b^{2}\right)}{8}-\frac{b^{4}}{2} \ln b \quad(>0), \\
f_{\mathrm{B}}(b, 0)=1,
\end{gathered}
$$

while in the special case of an inviscid surrounding fluid $(m \rightarrow \infty)$ equations (38)-(40) reduce to

$$
\begin{gathered}
f_{\mathrm{M}}(b, m)=-\frac{b^{3}}{2}\left(\frac{1-b^{2}}{1+b^{2}}+\ln b\right) \quad(>0), \\
f_{\mathrm{G}}(b, m)=-\frac{b^{4}}{2}\left(\frac{1-b^{2}}{1+b^{2}}+\ln b\right)=b f_{\mathrm{M}}(b, m) \quad(>0), \\
f_{\mathrm{B}}(b, m)=\frac{2 b^{2}}{1+b^{2}} \quad(>0),
\end{gathered}
$$

respectively. In addition, we note that $f_{\mathrm{M}}=-(1 / 2) b^{3} \ln b+O\left(b^{3}\right) \rightarrow 0^{+}, f_{\mathrm{G}}=-(1 / 2) b^{4} \ln b+O\left(b^{4}\right) \rightarrow 0^{+}$, and $f_{\mathrm{B}}=$ $2 b^{2}+O\left(b^{4}\right) \rightarrow 0^{+}$in the limit $b \rightarrow 0^{+}$, and that $f_{\mathrm{M}}=-(1-b)^{2}+O\left((1-b)^{3}\right) \rightarrow 0^{-}, f_{\mathrm{G}}=(2 / 3)(1-b)^{3}+O\left((1-b)^{4}\right) \rightarrow 0^{+}$, and $f_{\mathrm{B}}=1-4 m(1-b)^{2}+O\left((1-b)^{3}\right) \rightarrow 1^{-}$in the limit $b \rightarrow 1^{-}$.

As Figure 3 shows, the functions $f_{\mathrm{G}}$ and $f_{\mathrm{B}}$ are always positive, meaning that, as expected, the contributions to $Q_{1}$ and $-Q_{2}$ due to gravitational and background-flow effects are always of the same sign as $\Delta \rho$ and $Q_{\mathrm{b}}$, respectively, but the function $f_{\mathrm{M}}$ can be either positive, negative or zero, and so, despite the fact that $M$ is always non-negative, the contribution to $Q_{1}$ and $-Q_{2}$ due to Marangoni effects may be either positive, negative or zero. In particular, as 
Figure 3(a) also shows, there is a critical value of the droplet radius, denoted by $b_{\mathrm{c}}=b_{\mathrm{c}}(m)\left(0 \leq b_{\mathrm{c}} \leq 1\right)$, which depends on $m$ according to the equation

$$
f_{\mathrm{M}}\left(b_{\mathrm{c}}, m\right)=0,
$$

such that $f_{\mathrm{M}}$ is positive for $0<b<b_{\mathrm{c}}$ and negative for $b_{\mathrm{c}}<b<1$. As we shall see, the critical droplet radius $b_{\mathrm{c}}$ plays an important role in the motion of the droplet, and so Figure 4 shows $b_{\mathrm{c}}$ calculated from (47) plotted as a function of $m$. In particular, Figure 4 shows that $b_{\mathrm{c}}$ is a monotonically increasing function of $m$ satisfying $b_{\mathrm{c}} \sim \exp [-1 /(4 m)-1] \rightarrow 0^{+}$ in the limit $m \rightarrow 0^{+}$and $b_{\mathrm{c}} \sim 1-(3 / 2 m)^{1 / 2}+(5 / 4 m) \rightarrow 1^{-}$in the limit $m \rightarrow \infty$.

Now that the velocities, and hence the fluxes, are known, the evolution of the shape of the droplet is governed by the kinematic condition (31). The evolution of $b(t)$ and $L(t)$ is analysed in Section IV. Once the evolution of the shape of the droplet has been determined, we can determine the evolution of its position within the tube by considering the motion of the ends of the droplet. The evolution of $z_{\mathrm{f}}(t), z_{\mathrm{b}}(t)$ and $c(t)$ is analysed in Section $\mathrm{V}$.

\section{EVOLUTION OF THE SHAPE OF THE DROPLET}

Using (37), the kinematic condition (31) leads to the equation governing the evolution of the radius $b(t)$, and hence of the length $L(t)$, of the droplet, namely

$$
\frac{\mathrm{d} b}{\mathrm{~d} t}=-\frac{M f_{\mathrm{M}}(b, m)}{2 b},
$$

where $f_{\mathrm{M}}$ is given by (38). Equation (48) is a separable differential equation, with implicit solution $t=t(b)$ given by

$$
t=\frac{2}{M} \int_{b}^{b_{0}} \frac{\tilde{b}}{f_{\mathrm{M}}(\tilde{b}, m)} \mathrm{d} \tilde{b}
$$

where the initial value of $b$ is denoted by $b_{0}=b(0)$ (with the corresponding initial value of $L$ denoted by $L_{0}=L(0)=$ $\left.1 / b_{0}^{2}\right)$. However, since the integral in (48) cannot, in general, be evaluated in closed form, we investigated $b$ numerically via (48) and asymptotically via (49).

An important observation is that, since the gravitational and background-flow contributions to $Q_{1}$ and $-Q_{2}$ given by (37) are independent of $z$, they do not appear in (48), and hence the evolution of $b$ and $L$ is driven entirely by Marangoni effects (i.e. is independent of gravitational and background-flow effects). In particular, in the absence of Marangoni effects $(M=0)$, but not, in general, when they are present, $b$ and $L$ are constants (i.e. the droplet moves without changing shape). Moreover, since $M$ can be removed explicitly from (48) and (49) by an appropriate rescaling of time, changing the value of $M$ changes the timescale, but not the qualitative behaviour, of the evolution of $b$ and $L$.

Inspection of (48) with $f_{\mathrm{M}}$ given by (38) shows that, regardless of the values of $\Delta \rho$ and $Q_{\mathrm{b}}$, when $0<b<b_{\mathrm{c}}$ then $b$ always decreases and hence $L$ always increases in time, but when $b_{\mathrm{c}}<b<1$ then $b$ always increases and hence $L$ always decreases in time. In particular, this means that when the initial radius $b_{0}$ satisfies $0<b_{0}<b_{\mathrm{c}}$ the droplet becomes narrower and longer as time increases, but when it satisfies $b_{\mathrm{c}}<b_{0}<1$ the droplet becomes wider and shorter as time increases, ultimately filling the entire cross-section of the tube as $t \rightarrow \infty$. Specifically, equation (49) 

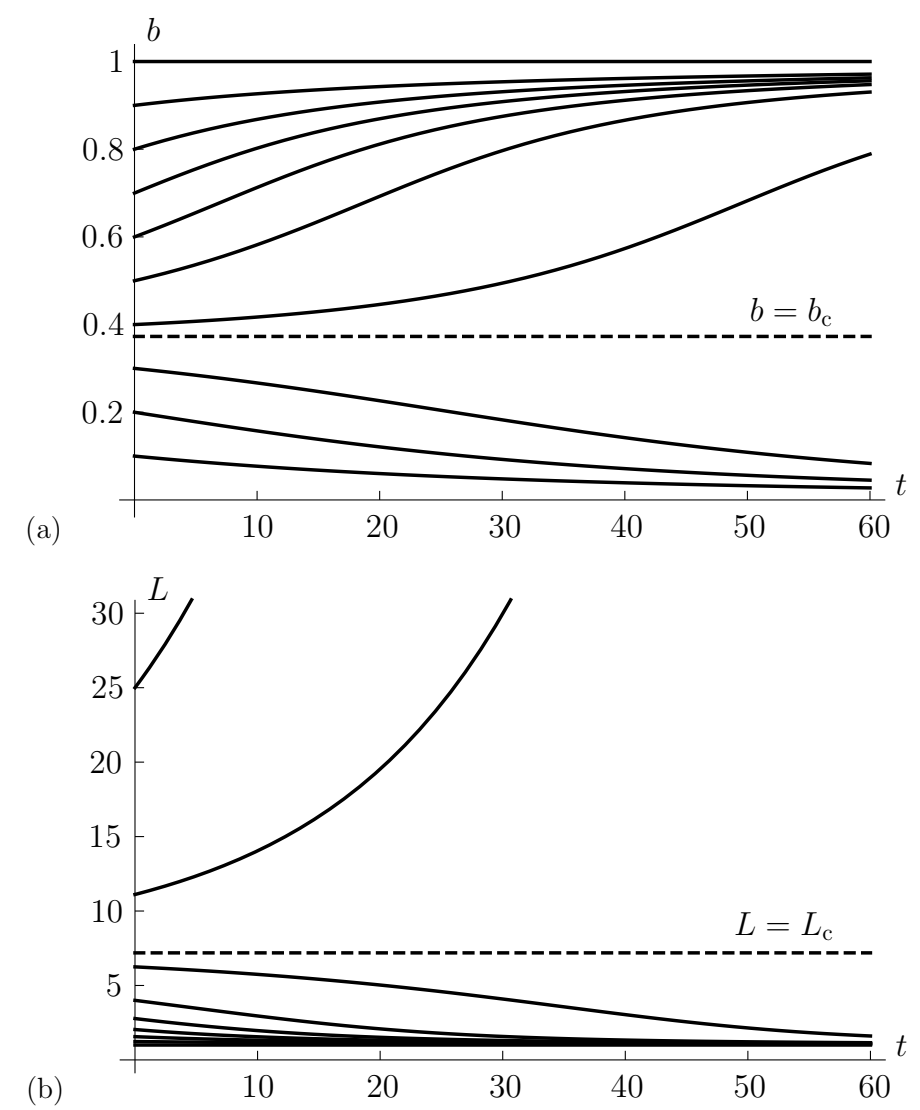

FIG. 5. Evolution of (a) the radius $b$ and (b) the length $L$ of the droplet calculated numerically from (48) for various initial radii $b_{0}$ and initial lengths $L_{0}=1 / b_{0}^{2}$ in the case $m=1$ and $M=1$. The critical values $b=b_{\mathrm{c}} \simeq 0.3729$ and $L=L_{\mathrm{c}}=1 / b_{\mathrm{c}}^{2} \simeq 7.1899$ are shown with dashed lines.

shows that

$$
b \sim b_{0}-\frac{M f_{\mathrm{M}}\left(b_{0}, m\right)}{2 b_{0}} t, \quad L \sim L_{0}+M f_{\mathrm{M}}\left(b_{0}, m\right) L_{0}^{2} t
$$

as $t \rightarrow 0^{+}$, and that

$$
b \sim \frac{4}{M t\left[\ln \frac{M t}{4}-\left(1+\frac{1}{4 m}\right)\right]} \rightarrow 0, \quad L \sim \frac{M^{2} t^{2}}{16}\left[\ln \frac{M t}{4}-\left(1+\frac{1}{4 m}\right)\right]^{2} \rightarrow \infty
$$

as $t \rightarrow \infty$ when $0<b_{0}<b_{\mathrm{c}}$, whereas

$$
b \sim 1-\frac{2}{M t} \rightarrow 1^{-}, \quad L \sim 1+\frac{4}{M t} \rightarrow 1^{+}
$$

as $t \rightarrow \infty$ when $b_{\mathrm{c}}<b_{0}<1$. In particular, in the special case of an inviscid bubble $(m=0)$ then $b_{\mathrm{c}}=0$ and hence the droplet always becomes wider and shorter, whereas in the special case of an inviscid surrounding fluid $(m \rightarrow \infty)$ then $b_{\mathrm{c}} \rightarrow 1^{-}$and hence the droplet always becomes narrower and longer.

Figure 5 shows the evolution of $b$ and $L$ calculated numerically from (48) for various $b_{0}$ and $L_{0}=1 / b_{0}^{2}$ in the case $m=1$ and $M=1$. In particular, Figure 5 illustrates how the critical values $b=b_{\mathrm{c}}$ and $L=L_{\mathrm{c}}=1 / b_{\mathrm{c}}^{2}$ separate droplets that become narrower and longer from those that become wider and shorter. 
At first sight, the fact that the droplet may either expand or contract might seem rather surprising as, regardless of the value of $m, \sigma$ is a quadratic function of $z$ given by (34), and hence Marangoni effects always drive flow away from $z=0$, and so we might naively expect the droplet always to become wider and shorter. However, while, as we have seen, this is true for an inviscid bubble with $m=0$, it is not, in general, true for a viscous droplet with $m \neq 0$, in which case the change in the shape of the droplet depends on the relative strengths of the Marangoni-induced flows in the two fluids. Specifically, for a droplet satisfying $0<b<b_{c}$ the flux of the Marangoni-driven axial flow in the droplet is a linearly increasing function of $z$, whereas that of the surrounding fluid is a linearly decreasing function of $z$, with the result that the droplet expands axially and contracts radially. On the other hand, for a droplet satisfying $b_{\mathrm{c}}<b<1$ the opposite occurs, with the result that the droplet contracts axially and expands radially.

Note that the behaviour described in this Section is qualitatively different from that for ordinary fluids for which $\sigma$ is a linearly decreasing function of $T$ analysed by Wilson [25]. In the latter case the Marangoni contribution to $Q_{1}$ and $-Q_{2}$ is independent of $z$, and hence $b$ and $L$ are constants (i.e. the droplet always moves without changing shape).

\section{EVOLUTION OF THE POSITION OF THE DROPLET}

As we showed in the previous Section, the present model predicts that the evolution of the shape of the droplet is driven entirely by Marangoni effects (i.e. is independent of gravitational and background-flow effects). However, as we shall now describe, the evolution of the position of the droplet depends, in general, on all three effects.

In order to determine the evolution of the position of the droplet we need to determine the evolution of the positions of the front, $z=z_{\mathrm{f}}(t)$ and the back, $z=z_{\mathrm{b}}(t)$, and hence of the centre of mass, $z=c(t)=\left(z_{\mathrm{f}}(t)+z_{\mathrm{b}}(t)\right) / 2$, of the droplet. In general, the details of the flow near the ends of the droplet will be complicated, but, since we are interested in the overall motion of the droplet rather than the details of the flow near its ends, a simple but realistic modelling assumption is that the motion of each end is due to the flux of fluid 1 at that end, i.e. we take the motion of the front and of the back of the droplet to be governed by

$$
b^{2} \frac{\mathrm{d} z_{\mathrm{f}}}{\mathrm{d} t}=\left.Q_{1}\right|_{z=z_{\mathrm{f}}(t)} \quad \text { and } \quad b^{2} \frac{\mathrm{d} z_{\mathrm{b}}}{\mathrm{d} t}=\left.Q_{1}\right|_{z=z_{\mathrm{b}}(t)},
$$

respectively. Adding together the two equations in (53) [41] yields

$$
b^{2} \frac{\mathrm{d} c}{\mathrm{~d} t}=\frac{1}{2}\left[\left.Q_{1}\right|_{z=z_{\mathrm{f}}(t)}+\left.Q_{1}\right|_{z=z_{\mathrm{b}}(t)}\right],
$$

which, since $Q_{1}$ given by (37) is a linear function of $z$, reduces to

$$
b^{2} \frac{\mathrm{d} c}{\mathrm{~d} t}=\left.Q_{1}\right|_{z=c(t)},
$$

leading to the equation governing the evolution of the centre of mass of the droplet, $c(t)$, namely

$$
\frac{\mathrm{d} c}{\mathrm{~d} t}=\frac{M c f_{\mathrm{M}}(b, m)+\Delta \rho f_{\mathrm{G}}(b, m)+Q_{\mathrm{b}} f_{\mathrm{B}}(b, m)}{b^{2}},
$$

where $f_{\mathrm{M}}, f_{\mathrm{G}}$ and $f_{\mathrm{B}}$ are given by (38), (39) and (40), respectively. With (48), equation (56) has a parametric solution 
for $c$ (with parameter $b$ ) given by

$$
c=\frac{b_{0}^{2} c_{0}}{b^{2}}-\frac{2}{b^{2}} \int_{b_{0}}^{b} \frac{\tilde{b}\left[\Delta \rho f_{\mathrm{G}}(\tilde{b}, m)+Q_{\mathrm{b}} f_{\mathrm{B}}(\tilde{b}, m)\right]}{M f_{\mathrm{M}}(\tilde{b}, m)} \mathrm{d} \tilde{b},
$$

where the initial value of $c$ is denoted by $c_{0}=c(0)$. However, as for (49), since the integral in (57) cannot, in general, be evaluated in closed form, we investigated $c$ numerically via (56) and asymptotically via (57). Once $c$ is known, the positions of the ends of the bubble are given simply by $z_{\mathrm{f}}=c+(L / 2)$ and $z_{\mathrm{f}}=c-(L / 2)$, respectively.

Equation (56) shows that, unlike the evolution of $b$ and $L$ described in Section IV, the evolution of $c$ depends on $\Delta \rho$ and $Q_{\mathrm{b}}$ as well as on $M$. In particular, equation (56) shows that, except in the case in which Marangoni effects are absent $(M=0)$ analysed in Section VI, in which case $b$ and $L$ and hence $\mathrm{d} c / \mathrm{d} t$ are constants, the velocity of the droplet, $\mathrm{d} c / \mathrm{d} t$, is not, in general, constant. Specifically, equation (57) shows that

$$
c \sim c_{0}+\frac{M c_{0} f_{\mathrm{M}}\left(b_{0}, m\right)+\Delta \rho f_{\mathrm{G}}\left(b_{0}, m\right)+Q_{\mathrm{b}} f_{\mathrm{B}}\left(b_{0}, m\right)}{b_{0}^{2}} t
$$

as $t \rightarrow 0^{+}$, and that the behaviour as $t \rightarrow \infty$ depends on whether $Q_{\mathrm{b}}$ is zero or non-zero.

In the special case of a closed tube with no background flow $Q_{\mathrm{b}}=0$ it is found from $(57)$ that when $0<b_{0}<b_{\mathrm{c}}$ then $c=O(t \ln t)^{2} \rightarrow \infty$ as $t \rightarrow \infty$ (i.e. that the droplet ultimately moves far away from $\left.z=0\right)$, but when $b_{\mathrm{c}}<b_{0}<1$ then $c$ approaches a constant value $c_{\infty}$ according to $c \sim c_{\infty}[1+4 /(M t)] \rightarrow c_{\infty}$ as $t \rightarrow \infty$ (i.e. that the droplet ultimately approaches a final stationary position).

In the general case $Q_{\mathrm{b}} \neq 0$ it is found from (57) that $|c| \rightarrow \infty$ as $t \rightarrow \infty$ (i.e. that the droplet again ultimately moves far away from $z=0)$. In particular, when $b_{\mathrm{c}}<b_{0}<1$ then $c / Q_{\mathrm{b}} \sim t+O(\ln t)$ as $t \rightarrow \infty$, showing that in this case the droplet ultimately moves at a constant velocity determined by the background flow. However, when $0<b_{0}<b_{\mathrm{c}}$ then the behaviour of $c$ has a more complicated dependence on $t$, and so in this case the droplet does not, in general, ultimately move with constant velocity.

In the next four Sections we use (56) and (57) to determine the evolution of $c$ in four different cases. Specifically, in Section VI we consider the case in which Marangoni effects are absent $(M=0)$, in Section VII we consider the case in which only Marangoni effects are present $\left(\Delta \rho=0\right.$ and $\left.Q_{\mathrm{b}}=0\right)$, while in Sections VIII and IX we consider the cases in which either only Marangoni effects and gravitational effects are present $\left(Q_{\mathrm{b}}=0\right)$ or only Marangoni effects and background-flow effects are present $(\Delta \rho=0)$, respectively. In each case, since the evolution of $b$ and $L$ has already been determined in Section IV, we need only determine the evolution of $c$ to complete the description of the motion of the droplet.

Note that, once again, the behaviour described in this Section is qualitatively different from that for ordinary fluids for which $\sigma$ is a linearly decreasing function of $T$ studied by Wilson [25]. In the latter case $b$ and $L$ are constants and the Marangoni contribution to $\mathrm{d} c / \mathrm{d} t$ is independent of $c$, and hence $\mathrm{d} c / \mathrm{d} t$ is constant (i.e. the velocity of the droplet is always constant). 


\section{MARANGONI EFFECTS ARE ABSENT $(M=0)$}

In the case in which Marangoni effects are absent $(M=0), b=b_{0}$ and $L=L_{0}$ are constants, and from (56) the droplet moves with constant velocity

$$
\frac{\mathrm{d} c}{\mathrm{~d} t}=\frac{\Delta \rho f_{\mathrm{G}}\left(b_{0}, m\right)+Q_{\mathrm{b}} f_{\mathrm{B}}\left(b_{0}, m\right)}{b_{0}^{2}}
$$

without changing shape. In particular,

$$
\frac{\mathrm{d} c}{\mathrm{~d} t}=\Delta \rho\left[\frac{\left(1-b_{0}^{2}\right)\left(1-3 b_{0}^{2}\right)}{8 b_{0}^{2}}-\frac{b_{0}^{2}}{2} \ln b_{0}\right]+\frac{Q_{\mathrm{b}}}{b_{0}^{2}}
$$

when $m=0$, and

$$
\frac{\mathrm{d} c}{\mathrm{~d} t}=-\frac{\Delta \rho b_{0}^{2}}{2}\left(\frac{1-b_{0}^{2}}{1+b_{0}^{2}}+\ln b_{0}\right)+\frac{2 Q_{\mathrm{b}}}{1+b_{0}^{2}}
$$

in the limit $m \rightarrow \infty$.

In this case we may, without loss of generality, take $\Delta \rho>0$, but $Q_{\mathrm{b}}$ may be positive, negative or zero. When $Q_{\mathrm{b}} \geq 0$ then background-flow and gravitational effects co-operate, and so $\mathrm{d} c / \mathrm{d} t>0$ (i.e. the droplet always moves upwards); however, when $Q_{\mathrm{b}}<0$ then the effects compete, and so $\mathrm{d} c / \mathrm{d} t$ may be positive, negative or zero (i.e. the droplet may move upwards, downwards or remain stationary).

Figure 6 shows $\mathrm{d} c / \mathrm{d} t$ plotted as a function of $b_{0}$ for the full range of values of $m$ for four different values of $Q_{\mathrm{b}}$ satisfying $Q_{\mathrm{b}}>0,-\Delta \rho / 8<Q_{\mathrm{b}}<0, Q_{\mathrm{b}}=-\Delta \rho / 8$ and $Q_{\mathrm{b}}<-\Delta \rho / 8$, respectively. In particular, Figure 6 illustrates that, except in the special case of an inviscid bubble $m=0$ [42], $\mathrm{d} c / \mathrm{d} t$ is a non-monotonic function of $b_{0}$, with either a maximum and a minimum (when $Q_{\mathrm{b}}<0$ ) or just a maximum (when $Q_{\mathrm{b}} \geq 0$ ) between its values $2 Q_{\mathrm{b}}$ and $Q_{\mathrm{b}}$ at $b_{0}=0$ and $b_{0}=1$, respectively. Intriguingly, Figure $6(\mathrm{~b})$ also illustrates that when $-\Delta \rho / 8<Q_{\mathrm{b}}<0$ droplets with radius $b_{0}=\left[1-\left(-8 Q_{\mathrm{b}} / \Delta \rho\right)^{1 / 2}\right]^{1 / 2}$ have the same velocity, namely $\mathrm{d} c / \mathrm{d} t=-\Delta \rho\left(1-b_{0}^{2}+2 b_{0}^{2} \ln b_{0}\right) / 4$, for all values of $m$.

\section{ONLY MARANGONI EFFECTS ARE PRESENT $\left(\Delta \rho=0\right.$ AND $\left.Q_{\mathrm{b}}=0\right)$}

In the case in which only Marangoni effects are present $\left(\Delta \rho=0\right.$ and $\left.Q_{\mathrm{b}}=0\right)$, then $b$ and $L$ evolve as described in Section IV, and from (57) the position of the centre of mass of the droplet is given by

$$
c=\frac{b_{0}^{2} c_{0}}{b^{2}}=b_{0}^{2} c_{0} L
$$

and hence the positions of the ends of the droplet are given by $z_{\mathrm{f}}=\left(b_{0}^{2} c_{0}+(1 / 2)\right) L$ and $z_{\mathrm{b}}=\left(b_{0}^{2} c_{0}-(1 / 2)\right) L$.

Figure 7 shows the evolution of $c$ for various $c_{0}$ for two different values of $b_{0}$, one satisfying $0<b_{0}<b_{\mathrm{c}}$ and the other satisfying $b_{\mathrm{c}}<b_{0}<1$. In particular, Figure 7(a) illustrates that when $0<b_{0}<b_{\mathrm{c}}$ then $c / c_{0}$ is an increasing function of $t$ satisfying $c / c_{0} \rightarrow \infty$ as $t \rightarrow \infty$ (i.e. the droplet always moves away from $z=0$ ), whereas when $b_{\mathrm{c}}<b_{0}<1$ then $c / c_{0}$ is a decreasing function of $t$ satisfying $c / c_{0} \rightarrow c_{\infty} / c_{0}=b_{0}^{2}(<1)$ as $t \rightarrow \infty$ (i.e. the droplet always moves towards $z=0$ ). Note that both parts of Figure 7 are symmetric about $c=0$, reflecting the symmetry of $\sigma$ given by (34) about $z=0$. 

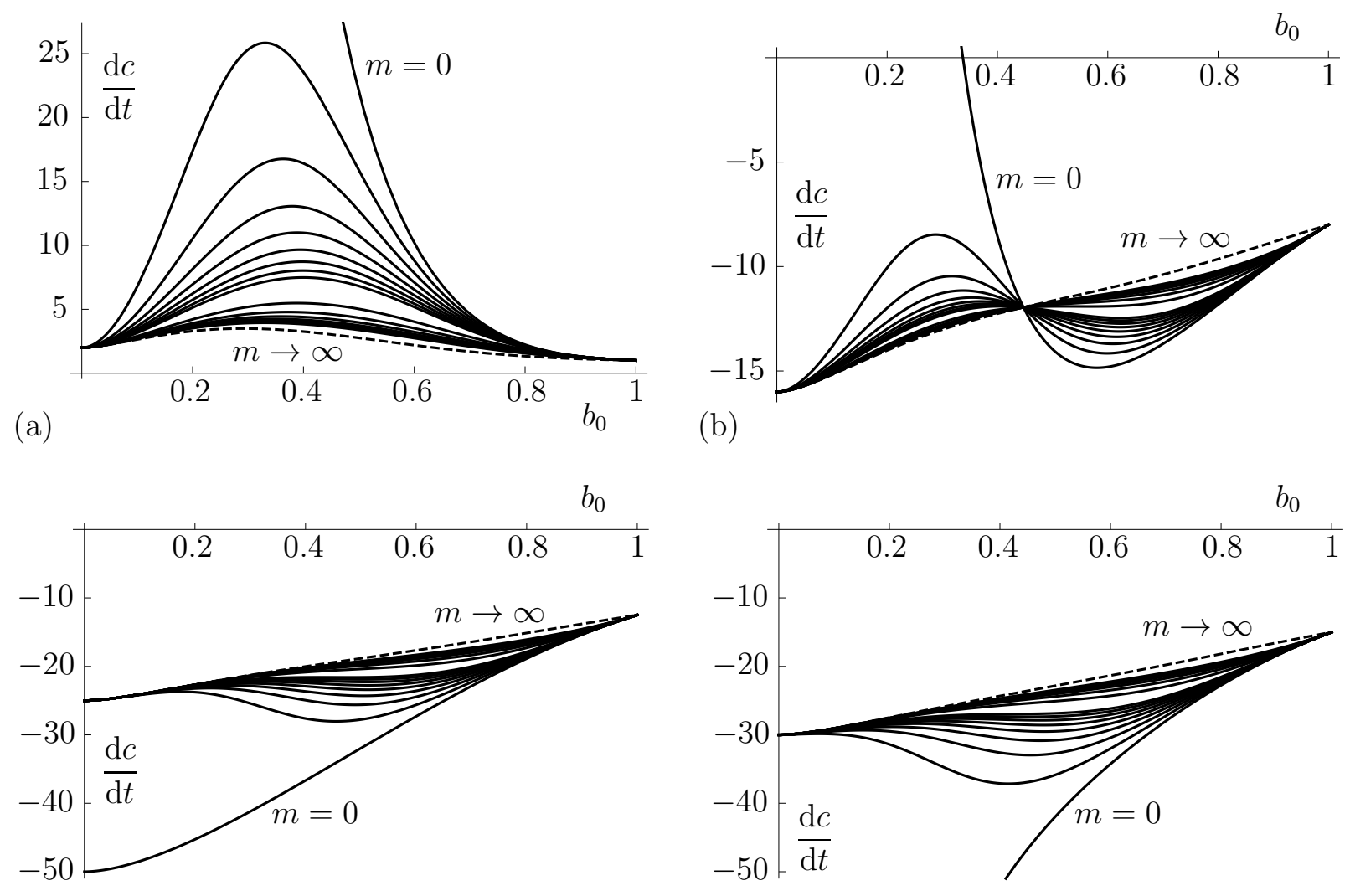

(c)

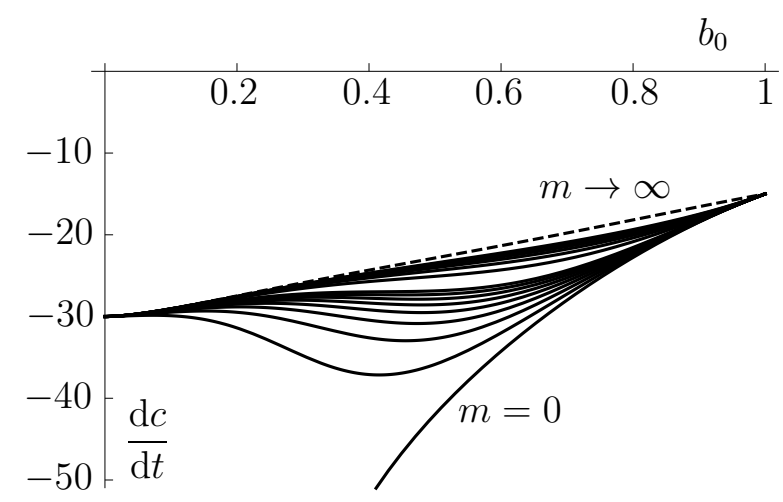

(d)

FIG. 6. Plots of the constant velocity of the droplet $\mathrm{d} c / \mathrm{d} t$ when Marangoni effects are absent given by (59) as a function of its initial radius $b_{0}$ in the case $M=0$ and $\Delta \rho=100$ when (a) $Q_{\mathrm{b}}=1$, (b) $Q_{\mathrm{b}}=-8$, (c) $Q_{\mathrm{b}}=-25 / 2$, and (d) $Q_{\mathrm{b}}=-15$ for $m=0,1 / 32,1 / 16, \ldots, 1 / 4,1 / 2, \ldots, 2$ and in the limit $m \rightarrow \infty$ given by (61) (the latter shown dashed).

Combining these results for the evolution of $c$ with those for the evolution of $b$ and $L$ described in Section IV, Figure 8 shows examples of the four qualitatively different forms of the evolution of a droplet when only Marangoni effects are present when $c_{0}>0$, illustrating that when $0<b_{0}<b_{\mathrm{c}}$ the droplet becomes narrower and longer and moves away from $z=0$ (in particular, satisfying $b \rightarrow 0^{+}, L \rightarrow \infty, c / c_{0} \rightarrow \infty$ as $t \rightarrow \infty$ ) as shown in Figures $8(\mathrm{a}, \mathrm{b})$, whereas when $b_{\mathrm{c}}<b_{0}<1$ the droplet becomes wider and shorter and moves towards $z=0$, ultimately filling the entire cross-section of the tube at a final stationary position which is closer to $z=0$ than its original position (in particular, satisfying $b \rightarrow 1^{-}, L \rightarrow 1^{+}$and $c / c_{0} \rightarrow c_{\infty} / c_{0}=b_{0}^{2}(<1)$ as $\left.t \rightarrow \infty\right)$ as shown in Figure 8(c,d). Note that in Figures 8(a,c) the entire droplet is initially (and always remains) above $z=0$, whereas in Figures $8(\mathrm{~b}, \mathrm{~d}$ ) the back of the droplet is initially (and always remains) below $z=0$. Because of the symmetry of $\sigma$ given by (34) about $z=0$, the corresponding evolutions when $c_{0}<0$ are simply the mirror images of those when $c_{0}>0$ in the plane $z=0$, and the evolutions when $c_{0}=0$ have $c \equiv 0$ for all $t$ (i.e. the droplet changes shape symmetrically with its centre of mass remaining stationary at $z=0$ ). 
(a)
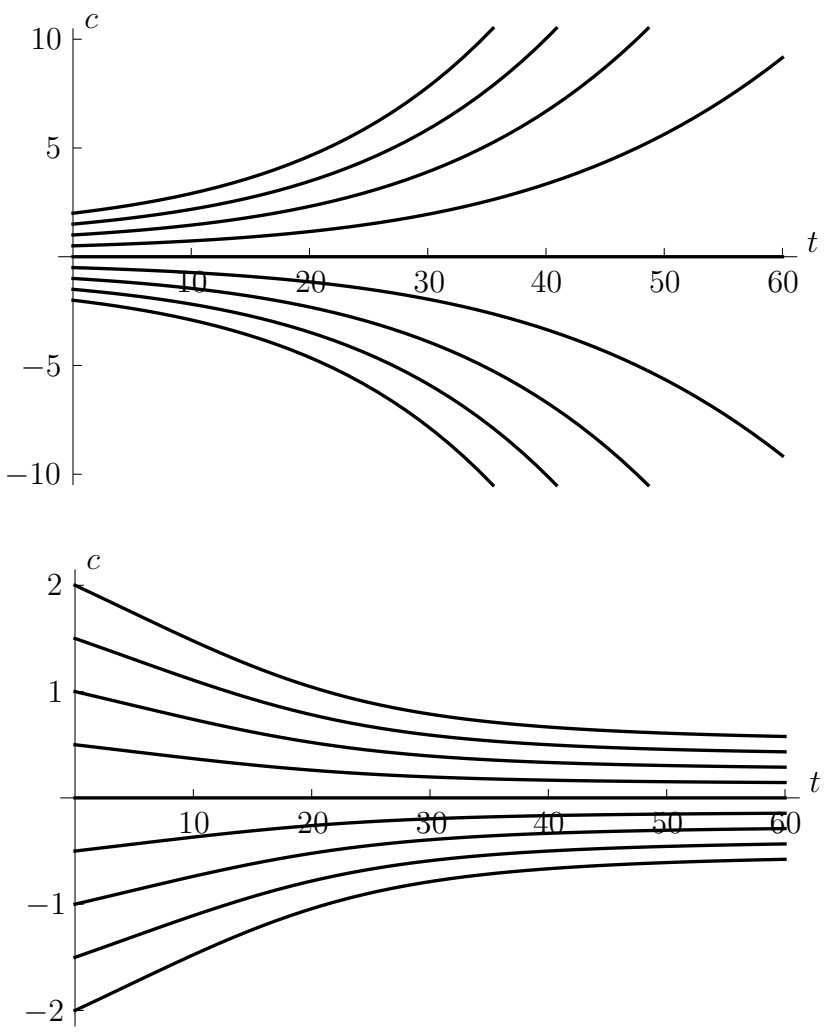

(b)

FIG. 7. Evolution of the position of the centre of mass of the droplet $c$ when only Marangoni effects are present given by (62) for various initial positions $c_{0}=-2,-3 / 2,-1, \ldots, 2$ in the case $m=1, M=1, \Delta \rho=0$ and $Q_{\mathrm{b}}=0$ when $(\mathrm{a}) b_{0}=1 / 4$ $\left(<b_{\mathrm{c}} \simeq 0.3729\right)$ and $(\mathrm{b}) b_{0}=1 / 2\left(>b_{\mathrm{c}}\right)$.

VIII. ONLY MARANGONI AND GRAVITATIONAL EFFECTS ARE PRESENT $\left(Q_{\mathrm{b}}=0\right)$

In the case in which only Marangoni and gravitational effects are present $\left(Q_{\mathrm{b}}=0\right)$, then $b$ and $L$ evolve as described in Section IV, and the position of the centre of mass of the droplet is given by (57).

In this case we may, without loss of generality, take $\Delta \rho>0$, and, like the case in which only Marangoni effects are present described in Section VII, despite the fact that $M$ is non-negative, the droplet may move upwards or downwards. However, in contrast to the case described in Section VII, in this case the droplet cannot remain stationary, and the competition between Marangoni and gravitational effects can lead to a no]n-monotonic evolution of $c$. In particular, recall from Section $\mathrm{V}$ that since $Q_{\mathrm{b}}=0$ then when $0<b_{0}<b_{\mathrm{c}}$ then $c=O(t \ln t)^{2} \rightarrow \infty$ as $t \rightarrow \infty$ (i.e. that the droplet ultimately moves far away from $z=0$ ), but when $b_{\mathrm{c}}<b_{0}<1$ then $c$ approaches a constant value $c_{\infty}$ according to $c \sim c_{\infty}[1+4 /(M t)] \rightarrow c_{\infty}$ as $t \rightarrow \infty$ (i.e. that the droplet ultimately approaches a final stationary position).

Figure 9 shows the evolution of $c$ for various $c_{0}$ for two different values of $b_{0}$, one satisfying $0<b_{0}<b_{\mathrm{c}}$ and the other satisfying $b_{\mathrm{c}}<b_{0}<1$.

Firstly, consider the case of a droplet satisfying $0<b_{0}<b_{\text {c }}$ shown in Figure 9 (a), for which, as we have already 
(a)

Hot

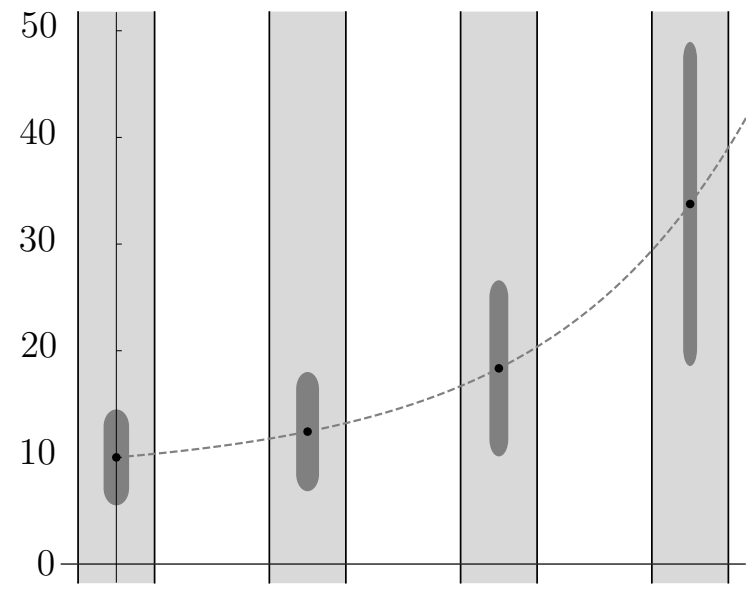

Cold

(c)

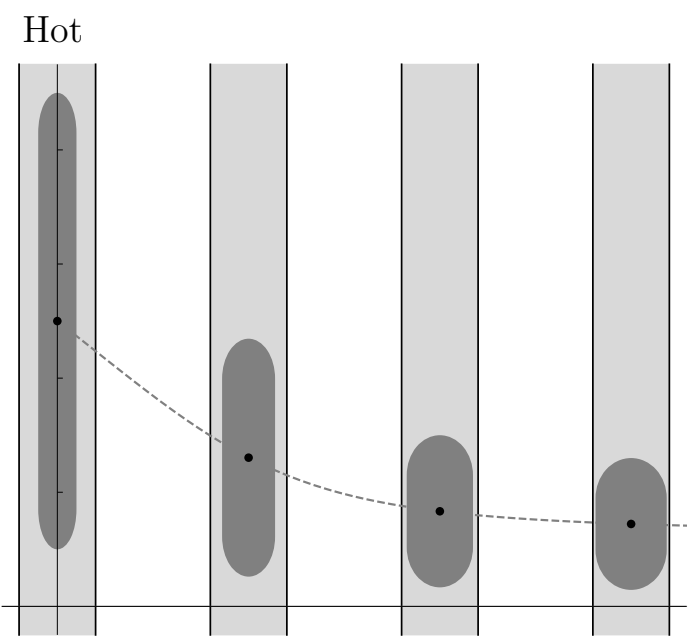

Cold (b)

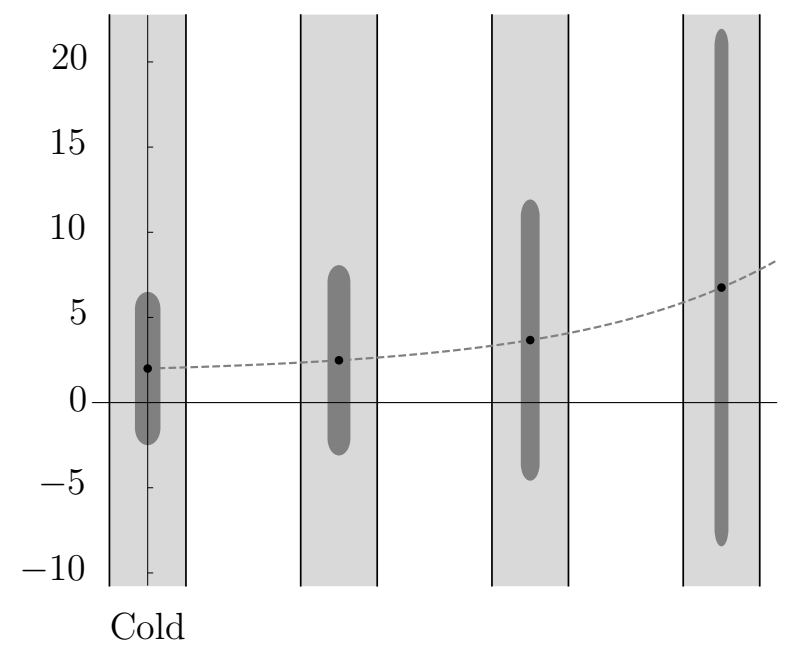

(d)

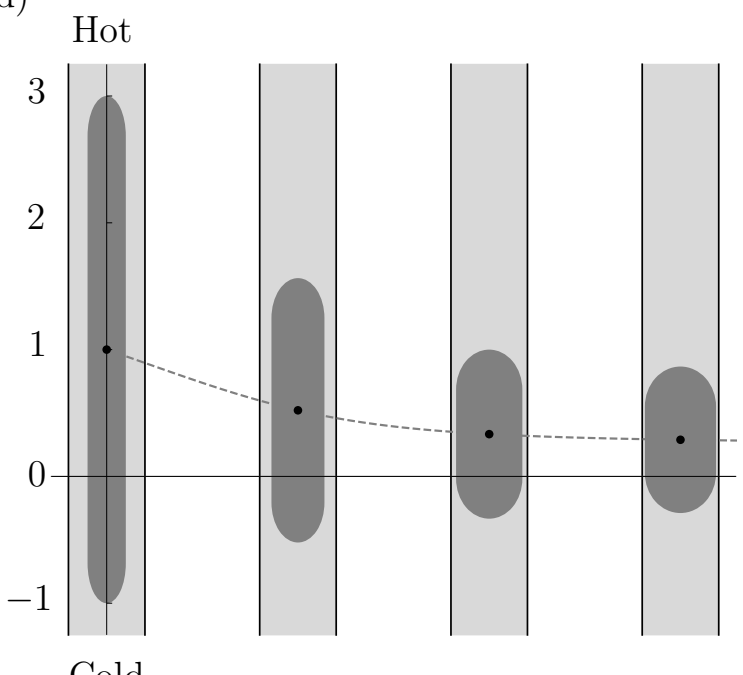

FIG. 8. Examples of the four qualitatively different forms of the evolution of a droplet when only Marangoni effects are present when $c_{0}>0$ in the case $m=1, M=1, \Delta \rho=0, Q_{\mathrm{b}}=0$, for which $b_{\mathrm{c}} \simeq 0.3729$. In (a) and (b), $L_{0}=9, b_{0}=1 / 3\left(<b_{\mathrm{c}}\right)$, with (a) $c_{0}=10$, (b) $c_{0}=2$, and the snapshots are shown at $t=0,15,30,45$, whereas in (c) and (d), $L_{0}=4, b_{0}=1 / 2\left(>b_{\mathrm{c}}\right)$, with (c) $c_{0}=5 / 2$, (d) $c_{0}=1$, and the snapshots are shown at $t=0,20,40,60$. In each snapshot the centre of mass of the droplet is denoted by a $\operatorname{dot}(\bullet)$. Note that the corresponding evolutions when $c_{0}<0$ are simply the mirror images of those when $c_{0}>0$ in the plane $z=0$, and the evolutions when $c_{0}=0$ have $c \equiv 0$ for all $t$.

described in Section IV, $b \rightarrow 0^{+}$and $L \rightarrow \infty$ as $t \rightarrow \infty$. In this case (57) shows that $|c| \rightarrow \infty$ according to

$$
c \sim \frac{k}{b^{2}}
$$

as $t \rightarrow \infty$, where

$$
k=b_{0}^{2} c_{0}+\frac{2 \Delta \rho}{M} \int_{0}^{b_{0}} \frac{b f_{\mathrm{G}}(b, m)}{f_{\mathrm{M}}(b, m)} \mathrm{d} b
$$


(a)
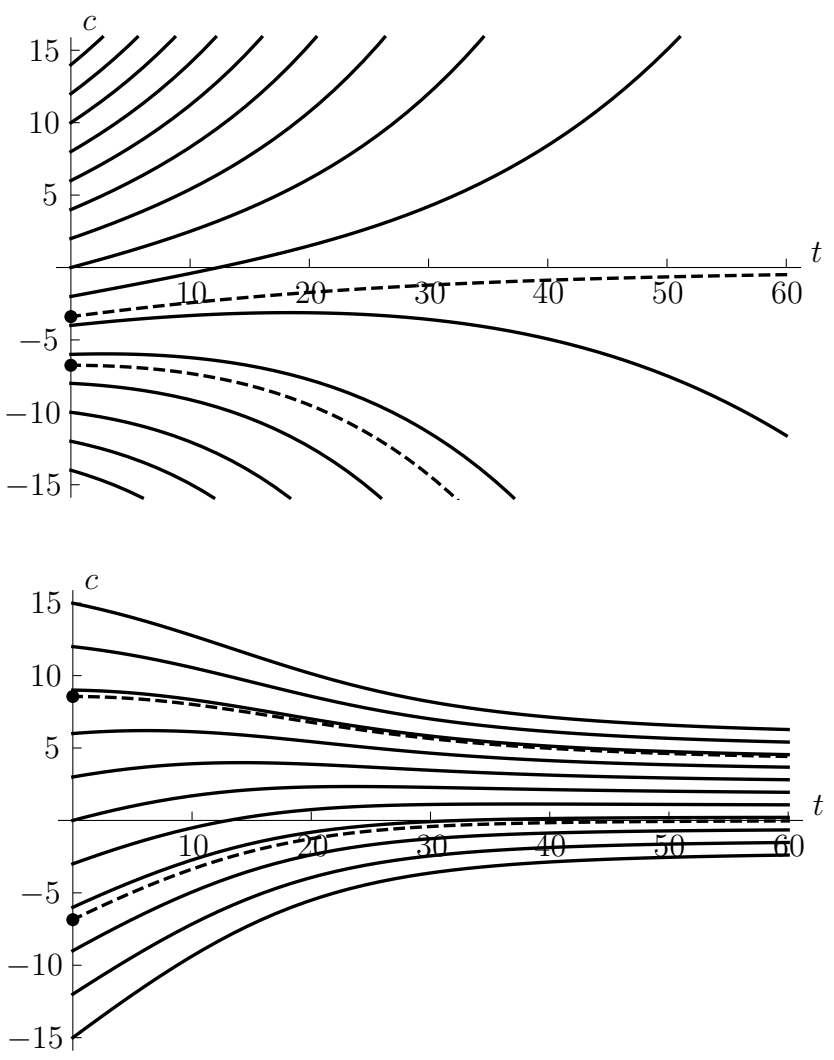

(b)

FIG. 9. Evolution of the position of the centre of mass of the droplet $c$ when only Marangoni and gravitational effects are present given by (57) in the case $m=1, M=1, \Delta \rho=10$ and $Q_{\mathrm{b}}=0$ for initial positions (a) $c_{0}=-14,-12,-10, \ldots, 12,14$ when $b_{0}=1 / 4\left(<b_{\mathrm{c}} \simeq 0.3729\right)$ and (b) $c_{0}=-15,-12,-9, \ldots, 12,15$ when $b_{0}=1 / 2\left(>b_{\mathrm{c}}\right)$. The evolutions corresponding to the critical values $(\mathrm{a}) c_{0}=c_{1}^{\mathrm{G}} \simeq-6.7523$ and $c_{0}=c_{2}^{\mathrm{G}} \simeq-3.3930$, and $(\mathrm{b}) c_{0}=c_{1}^{\mathrm{G}} \simeq 8.5571$ and $c_{0}=c_{2}^{\mathrm{G}} \simeq-6.8558$, which separate the three different behaviours, are shown with dashed lines.

is a constant. In particular, Figure 9(a) shows that there are three qualitatively different possible evolutions of $c$ depending on the value of $c_{0}$ in relation to two critical values, denoted by $c_{1}^{\mathrm{G}}$ and $c_{2}^{\mathrm{G}}$, where $c_{1}^{\mathrm{G}}<c_{2}^{\mathrm{G}}$. Specifically, when $c_{0}>c_{2}^{\mathrm{G}}$ then $c$ increases monotonically, when $c_{1}^{\mathrm{G}}<c_{0}<c_{2}^{\mathrm{G}}$ then $c$ first increases to a maximum and then decreases, and when $c_{0}<c_{1}^{\mathrm{G}}$ then $c$ decreases monotonically, where the critical value $c_{1}^{\mathrm{G}}$ is the value of $c_{0}$ for which $\mathrm{d} c / \mathrm{d} t(0)=0$, which from (58) is given by

$$
c_{1}^{\mathrm{G}}=-\frac{\Delta \rho f_{\mathrm{G}}\left(b_{0}, m\right)}{M f_{\mathrm{M}}\left(b_{0}, m\right)},
$$

and the critical value $c_{2}^{\mathrm{G}}$ is the value of $c_{0}$ for which $k=0$, namely

$$
c_{2}^{\mathrm{G}}=-\frac{2 \Delta \rho}{M b_{0}^{2}} \int_{0}^{b_{0}} \frac{b f_{\mathrm{G}}(b, m)}{f_{\mathrm{M}}(b, m)} \mathrm{d} b .
$$

Secondly, consider the case of a droplet satisfying $b_{\mathrm{c}}<b_{0}<1$ shown in Figure $9(\mathrm{~b})$, for which, as we have already described in Section IV, $b \rightarrow 1^{-}$and $L \rightarrow 1^{+}$as $t \rightarrow \infty$. In this case (57) shows that $c \rightarrow c_{\infty}$ as $t \rightarrow \infty$, where

$$
c_{\infty}=b_{0}^{2} c_{0}-\frac{2 \Delta \rho}{M} \int_{b_{0}}^{1} \frac{b f_{\mathrm{G}}(b, m)}{f_{\mathrm{M}}(b, m)} \mathrm{d} b .
$$


Specifically, Figure 9(b) shows that there are again three qualitatively different possible evolutions of $c$ depending on the value of $c_{0}$ in relation to two critical values, again denoted by $c_{1}^{\mathrm{G}}$ and $c_{2}^{\mathrm{G}}$, where now $c_{1}^{\mathrm{G}}>c_{2}^{\mathrm{G}}$. Specifically, when $c_{0}>c_{1}^{\mathrm{G}}$ then $c$ decreases monotonically, when $c_{2}^{\mathrm{G}}<c_{0}<c_{1}^{\mathrm{G}}$ then $c$ first increases to a maximum and then decreases,

and when $c_{0}<c_{2}^{\mathrm{G}}$ then $c$ increases monotonically, where the critical value $c_{1}^{\mathrm{G}}$ is again given by (65) but the critical value $c_{2}^{\mathrm{G}}$ is now the value of $c_{0}$ for which $c_{\infty}=0$, namely

$$
c_{2}^{\mathrm{G}}=\frac{2 \Delta \rho}{M b_{0}^{2}} \int_{b_{0}}^{1} \frac{b f_{\mathrm{G}}(b, m)}{f_{\mathrm{M}}(b, m)} \mathrm{d} b .
$$

\section{ONLY MARANGONI AND BACKGROUND-FLOW EFFECTS ARE PRESENT $(\Delta \rho=0)$}

In the case in which only Marangoni and background-flow effects are present $(\Delta \rho=0)$, then $b$ and $L$ evolve as described in Section IV, and the position of the centre of mass of the droplet is given by (57).

In this case we may, without loss of generality, take $Q_{\mathrm{b}}>0$, and, like the case in which Marangoni and gravitational effects are present described in Section VIII, despite the fact that $M$ is non-negative, the droplet may again move upwards or downwards. Moreover, like in the case described in Section VIII, in this case the droplet cannot remain stationary, and the competition between Marangoni and background-flow effects can lead to a similar (but not exactly equivalent) non-monotonic evolution of $c$. In particular, recall from Section $\mathrm{V}$ that since $Q_{\mathrm{b}} \neq 0$ then $|c| \rightarrow \infty$ as $t \rightarrow \infty$ (i.e. that the droplet ultimately moves far away from $z=0$ ).

Figure 10 shows typical evolutions of $c$ for various $c_{0}$ for two different values of $b_{0}$, one satisfying $0<b_{0}<b_{\mathrm{c}}$ and the other satisfying $b_{\mathrm{c}}<b_{0}<1$.

Firstly, consider the case of a droplet satisfying $0<b_{0}<b_{\mathrm{c}}$ shown in Figure 10(a). The evolution of $c$ in this case parallels that shown in Figure $9(\mathrm{a})$ (with $\Delta \rho, f_{\mathrm{G}}, c_{1}^{\mathrm{G}}$ and $c_{2}^{\mathrm{G}}$ replaced with $Q_{\mathrm{b}}, f_{\mathrm{B}}, c_{1}^{\mathrm{B}}$ and $c_{2}^{\mathrm{B}}$, respectively), and so the discussion need not be repeated here. The critical values $c_{1}^{\mathrm{B}}$ and $c_{2}^{\mathrm{B}}$, where $c_{1}^{\mathrm{B}}<c_{2}^{\mathrm{B}}$, are given by

$$
c_{1}^{\mathrm{B}}=-\frac{Q_{\mathrm{b}} f_{\mathrm{B}}\left(b_{0}, m\right)}{M f_{\mathrm{M}}\left(b_{0}, m\right)}
$$

and

$$
c_{2}^{\mathrm{B}}=-\frac{2 Q_{\mathrm{b}}}{M b_{0}^{2}} \int_{0}^{b_{0}} \frac{b f_{\mathrm{B}}(b, m)}{f_{\mathrm{M}}(b, m)} \mathrm{d} b
$$

Secondly, consider the case of a droplet satisfying $b_{\mathrm{c}}<b_{0}<1$ shown in Figure 10(b). In this case (57) shows that $c \sim Q_{\mathrm{b}} t \rightarrow \infty$ as $t \rightarrow \infty$, and the evolution of $c$ in this case is similar to, but qualitatively different from, that shown in Figure 9(b). Specifically, when $c_{0}>c_{1}^{\mathrm{B}}$ then $c$ first decreases and then increases with $t$, when $c_{2}^{\mathrm{B}}<c_{0}<c_{1}^{\mathrm{B}}$ then $c$ first increases, then decreases, and then increases again, and when $c_{0}<c_{2}^{\mathrm{B}}$ then $c$ increases monotonically with $t$. The critical value $c_{1}^{\mathrm{B}}$ is defined in the same way that $c_{1}^{\mathrm{G}}$ was in Section VIII, and so is again given by (69). However, since there is now no value of $c_{0}$ that leads to a constant value of $c$ as $t \rightarrow \infty$, the critical value $c_{2}^{\mathrm{B}}$ is not defined in the way that $c_{2}^{\mathrm{G}}$ was in Section VIII. Instead, $c_{2}^{\mathrm{B}}$ is determined as the value of $c_{0}$ for which the maximum, the minimum, and the point of inflection of the solution $c$ coincide, so that $\mathrm{d} c / \mathrm{d} t=0$ and $\mathrm{d}^{2} c / \mathrm{d} t^{2}=0$. With (48) these lead to the condition

$$
f_{\mathrm{B}} \frac{\partial f_{\mathrm{M}}}{\partial b}-f_{\mathrm{M}} \frac{\partial f_{\mathrm{B}}}{\partial b}=0
$$


(a)
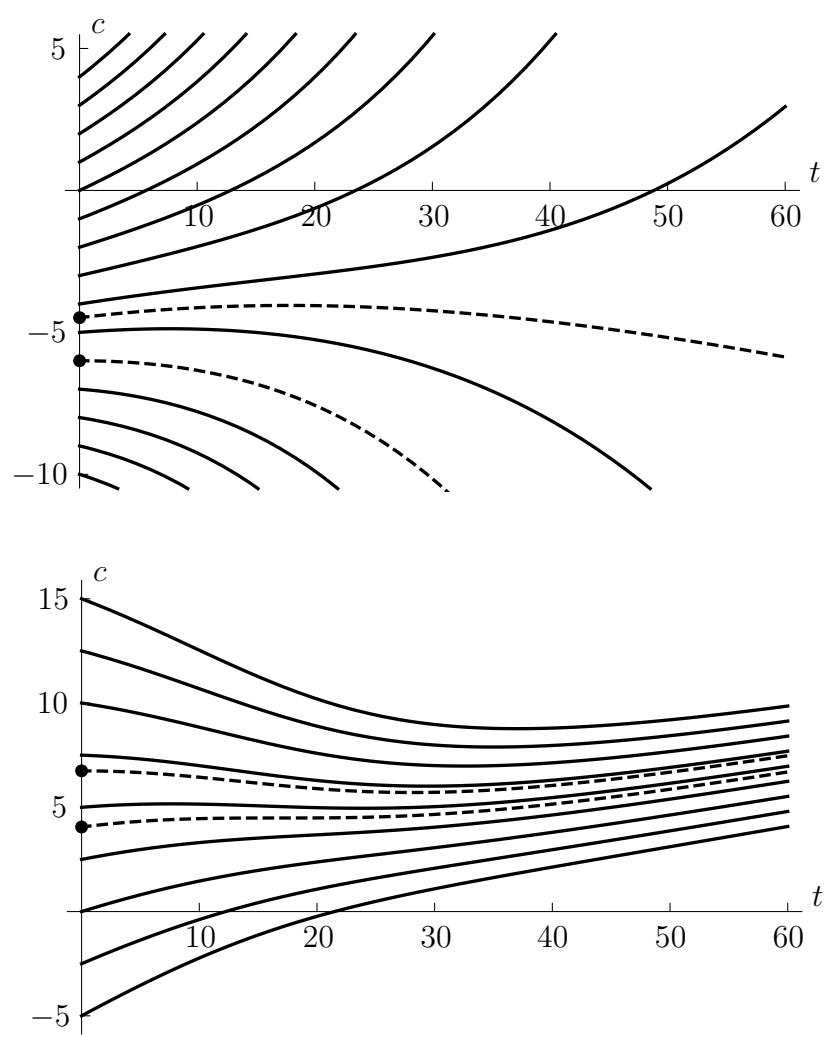

(b)

FIG. 10. Evolution of the position of the centre of mass of the droplet $c$ when only Marangoni and background-flow effects are present given by (57) in the case $m=1, M=1, \Delta \rho=0$ and $Q_{\mathrm{b}}=1 / 10$ for initial positions (a) $c_{0}=-10,-9,-8, \ldots, 3,4$ when $b_{0}=1 / 4\left(<b_{\mathrm{c}} \simeq 0.3729\right)$ and $(\mathrm{b}) c_{0}=-5,-5 / 2,0, \ldots, 25 / 2,15$ when $b_{0}=1 / 2\left(>b_{\mathrm{c}}\right)$. The evolutions corresponding to the critical values $(\mathrm{a}) c_{0}=c_{1}^{\mathrm{B}} \simeq-5.9993$ and $c_{0}=c_{2}^{\mathrm{B}} \simeq-4.4817$, and (b) $c_{0}=c_{1}^{\mathrm{B}} \simeq 6.7484$ and $c_{0}=c_{2}^{\mathrm{B}} \simeq 4.0561$, which separate the three different behaviours, are shown with dashed lines.

determining the value $b=b_{\mathrm{SP}}$ of $b$ corresponding to the stationary value $c=c_{\mathrm{SP}}$ of $c$. Equation (56) then gives $c_{\mathrm{SP}}=-Q_{\mathrm{b}} f_{\mathrm{B}} / M f_{\mathrm{M}}$ evaluated at $b=b_{\mathrm{SP}}$, and so $c_{2}^{\mathrm{B}}$ is obtained from (57) as

$$
c_{2}^{\mathrm{B}}=\left.\frac{Q_{\mathrm{b}}}{M b_{0}^{2}}\left(2 \int_{b_{0}}^{b} \frac{\tilde{b} f_{\mathrm{B}}}{f_{\mathrm{M}}} \mathrm{d} \tilde{b}-\frac{b^{2} f_{\mathrm{B}}}{f_{\mathrm{M}}}\right)\right|_{b=b_{\mathrm{SP}}} .
$$

\section{CONCLUSIONS}

In the present work we formulated and analysed a theoretical model for the unsteady motion of a long bubble or droplet in a self-rewetting system in a non-uniformly heated tube due to a combination of Marangoni effects, gravitational effects due to the density difference between the two fluids, and an imposed background flow along the tube.

We found that the present model predicts that the evolution of the shape of the droplet is driven entirely by Marangoni effects, and depends on the initial value of its radius $b_{0}$ in relation to the critical value $b_{\mathrm{c}}=b_{\mathrm{c}}(m)$ given by (47). Specifically, as described in Section IV, when $0<b_{0}<b_{\mathrm{c}}$ the droplet becomes narrower and longer as time 
increases, but when $b_{\mathrm{c}}<b_{0}<1$ the droplet becomes wider and shorter as time increases, ultimately filling the entire cross-section of the tube as $t \rightarrow \infty$. However, as described in Section V, the evolution of the position of the droplet depends, in general, on gravitational and background-flow effects in addition to Marangoni effects. In the case in which Marangoni effects are absent the droplet always moves with constant velocity given by (59) without changing shape. In the case in which only Marangoni effects are present when $0<b_{0}<b_{\mathrm{c}}$ the droplet always moves away from $z=0$ as time increases, but when $b_{\mathrm{c}}<b_{0}<1$ the droplet moves towards $z=0$ as time increases, ultimately filling the entire cross-section of the tube at a final stationary position which is closer to $z=0$ than its original position. Figure 8 shows examples of the four qualitatively different forms of the evolution of the droplet in this case. In the cases in which either only Marangoni effects and gravitational effects or only Marangoni effects and background-flow effects are present the competition between the two effects can lead to a non-monotonic evolution of $c$.

For completeness, the corresponding results for the closely related problem of two-dimensional droplet in a channel are presented in Appendix B.

Finally, it should be re-iterated that the behaviour of a self-rewetting system described in the present work is qualitatively different from that for ordinary fluids, in which case the droplet always moves with constant velocity without changing shape.

\section{ACKNOWLEDGEMENTS}

All four authors gratefully acknowledge the financial support provided by The Carnegie Trust for the Universities of Scotland via Research Incentive Grant (RIG) 70775 "Mathematical Modelling and Analysis of Self-Rewetting Fluids in Heat Pipes". KS also acknowledges the financial support of the Engineering and Physical Sciences Research Council (EPSRC) via research grant EP/N011341/1 "Flow Boiling and Condensation of Mixtures in Microscale".

[1] R. Vochten and G. Petre, Study of the heat of reversible adsorption at the air-solution interface. II. Experimental determination of the heat of reversible adsorption of some alcohols, Journal of Colloid and Interface Science 42, 320-327 (1973).

[2] G. Petre and M. A. Azouni, Experimental evidence for the minimum of surface tension with temperature at aqueous alcohol solution/air interfaces, Journal of Colloid and Interface Science 98, 261-263 (1984).

[3] J. C. Legros, Problems related to non-linear variations of surface tension, Acta Astronautica 13, 697-703 (1986).

[4] G. Vázquez, E. Alvarez, and J. M. Navaza, Surface tension of alcohol + water from 20 to $50{ }^{\circ} \mathrm{C}$, Journal of Chemical and Engineering Data 40, 611-614 (1995).

[5] N. Zhang, Innovative heat pipe systems using a new working fluid, International Communications in Heat and Mass Transfer 28, 1025-1033 (2001).

[6] Y. Abe, A. Iwasaki, and K. Tanaka, Microgravity experiments on phase change in self-rewetting fluids, Annals of the New York Academy of Sciences 1027, 269-285 (2004).

[7] Y. Abe, A. Iwasaki, and K. Tanaka, Thermal management with self-rewetting fluids, Microgravity Science and Technology 16, 148-152 (2005). 
[8] Y. Abe, Self-rewetting fluids, Annals of the New York Academy of Sciences 1077, 650-667 (2006).

[9] R. Savino and D. Paterna, Marangoni effect and heat pipe dry-out, Physics of Fluids 18, 118103 (2006).

[10] R. Savino, N. di Francescantonio, R. Fortezza, and Y. Abe, Heat pipes with binary mixtures and inverse Marangoni effects for microgravity applications, Acta Astronautica 61, 16-26 (2007).

[11] R. Savino, C. Piccolo, R. Fortezza, and Y. Abe, Heat pipes with self-rewetting fluids in low-gravity conditions, Microgravity Science and Technology 19, 75-77 (2007).

[12] N. di Francescantonio, R. Savino, and Y. Abe, New alcohol solutions for heat pipes: Marangoni effect and heat transfer enhancement, International Journal of Heat and Mass Transfer 51, 6199-6207 (2008).

[13] R. Savino, A. Cecere, and R. Di Paola, Surface tension-driven flow in wickless heat pipes with self-rewetting fluids, International Journal of Heat and Fluid Flow 30, 380-388 (2009).

[14] R. Savino, R. Di Paola, A. Cecere, and R. Fortezza, Self-rewetting heat transfer fluids and nanobrines for space heat pipes, Acta Astronautica 67, 1030-1037 (2010).

[15] R. Savino, D. De Cristofaro, and A. Cecere, Flow visualization and analysis of self-rewetting fluids in a model heat pipe, International Journal of Heat and Mass Transfer 115, 581-591 (2017).

[16] Y. Hu, T. Liu, X. Li, and S. Wang, Heat transfer enhancement of micro oscillating heat pipes with self-rewetting fluid, International Journal of Heat and Mass Transfer 70, 496-503 (2014).

[17] Y. Hu, S. Zhang, X. Li, and S. Wang, Heat transfer enhancement of subcooled pool boiling with self-rewetting fluid, International Journal of Heat and Mass Transfer 83, 64-68 (2015).

[18] S.-C. Wu, Study of self-rewetting fluid applied to loop heat pipe, International Journal of Thermal Sciences 98, 374-380 (2015).

[19] A. Oron and P. Rosenau, On a nonlinear thermocapillary effect in thin liquid layers, Journal of Fluid Mechanics 273, 361-374 (1994).

[20] S. G. Slavtchev and S. P. Miladinova, Thermocapillary flow in a liquid layer at minimum in surface tension, Acta Mechanica 127, 209-224 (1998).

[21] W. Batson, Y. Agnon, and A. Oron, Thermocapillary modulation of self-rewetting films, Journal of Fluid Mechanics 819, 562-591 (2017).

[22] N. O. Young, J. S. Goldstein, and M. J. Block, The motion of bubbles in a vertical temperature gradient, Journal of Fluid Mechanics 6, 350-356 (1959).

[23] R. Balasubramaniam and A.-T. Chai, Thermocapillary migration of droplets: an exact solution for small Marangoni numbers, Journal of Colloid and Interface Science 119, 531-538 (1987).

[24] P. Ehrhard and S. H. Davis, Non-isothermal spreading of liquid drops on horizontal plates, Journal of Fluid Mechanics 229, 365-388 (1991).

[25] S. K. Wilson, The steady thermocapillary-driven motion of a large droplet in a closed tube, Physics of Fluids A: Fluid Dynamics 5, 2064-2066 (1993).

[26] S. K. Wilson, The effect of an axial temperature gradient on the steady motion of a large droplet in a tube, Journal of Engineering Mathematics 29, 205-217 (1995).

[27] M. K. Smith, Thermocapillary migration of a two-dimensional liquid droplet on a solid surface, Journal of Fluid Mechanics 294, 209-230 (1995).

[28] R. Balasubramaniam and R. S. Subramanian, The migration of a drop in a uniform temperature gradient at large Marangoni numbers, Physics of Fluids 12, 733-743 (2000).

[29] A. Mazouchi and G. M. Homsy, Thermocapillary migration of long bubbles in cylindrical capillary tubes, Physics of Fluids 12, 542-549 (2000). 
[30] A. Mazouchi and G. M. Homsy, Thermocapillary migration of long bubbles in polygonal tubes. I. Theory, Physics of Fluids 13, 1594-1600 (2001).

[31] E. Lajeunesse and G. M. Homsy, Thermocapillary migration of long bubbles in polygonal tubes. II. Experiments, Physics of Fluids 15, 308-314 (2003).

[32] G. J. Dunn, B. R. Duffy, S. K. Wilson, and D. Holland, Quasi-steady spreading of a thin ridge of fluid with temperaturedependent surface tension on a heated or cooled substrate, Quarterly Journal of Mechanics and Applied Mathematics 62, 365-402 (2009).

[33] E. Katz, M. Haj, A. M. Leshansky, and A. Nepomnyashchy, Thermocapillary motion of a slender viscous droplet in a channel, Physics of Fluids 24, 022102 (2012).

[34] G. Karapetsas, K. C. Sahu, and O. K. Matar, Effect of contact line dynamics on the thermocapillary motion of a droplet on an inclined plate, Langmuir 29, 8892-8906 (2013).

[35] G. Karapetsas, K. C. Sahu, K. Sefiane, and O. K. Matar, Thermocapillary-driven motion of a sessile drop: effect of non-monotonic dependence of surface tension on temperature, Langmuir 30, 4310-4321 (2014).

[36] M. K. Tripathi, K. C. Sahu, G. Karapetsas, K. Sefiane, and O. K. Matar, Non-isothermal bubble rise: non-monotonic dependence of surface tension on temperature, Journal of Fluid Mechanics 763, 82-108 (2015).

[37] M. E. R. Shanahan and K. Sefiane, Recalcitrant bubbles, Scientific Reports 4, 4727 (2014).

[38] D. Mamalis, V. Koutsos, and K. Sefiane, On the motion of a sessile drop on an incline: effect of non-monotonic thermocapillary stresses, Applied Physics Letters 109, 231601 (2016).

[39] D. Mamalis, V. Koutsos, and K. Sefiane, Bubble rise in a non-isothermal self-rewetting fluid and the role of thermocapillarity, International Journal of Thermal Sciences 117, 146-162 (2017).

[40] D. Mamalis, V. Koutsos, and K. Sefiane, Nonisothermal spreading dynamics of self-rewetting droplets, Langmuir 34, 1916-1931 (2018).

[41] Note that subtracting the second of equations in (53) from the first yields

$$
b^{2} \frac{\mathrm{d} L}{\mathrm{~d} t}=\left.Q_{1}\right|_{z=z_{\mathrm{f}}(t)}-\left.Q_{1}\right|_{z=z_{\mathrm{b}}(t)}=M f_{\mathrm{M}}\left(z_{\mathrm{f}}-z_{\mathrm{b}}\right)=M f_{\mathrm{M}} L=-2 b L \frac{\mathrm{d} b}{\mathrm{~d} t},
$$

so that $\mathrm{d}\left(b^{2} L\right) / \mathrm{d} t=0$, which recovers the volume-conservation condition $b^{2} L=1$, but provides no new information.

[42] Figure 6 also illustrates that in the special case of an inviscid bubble $m=0$ given by (60), d $c / \mathrm{d} t$ decreases monotonically with $b_{0}$ when $Q_{\mathrm{b}} \geq 0$, has a minimum value at some $b_{0}$ when $-\Delta \rho / 8<Q_{\mathrm{b}}<0$, and increases monotonically with $b_{0}$ when $Q_{\mathrm{b}} \leq-\Delta \rho / 8$, with $\mathrm{d} c / \mathrm{d} t \rightarrow \infty$ when $Q_{\mathrm{b}}>-\Delta \rho / 8, \mathrm{~d} c / \mathrm{d} t \rightarrow 4 Q_{\mathrm{b}}=-\Delta \rho / 2$ when $Q_{\mathrm{b}}=-\Delta \rho / 8$, and $\mathrm{d} c / \mathrm{d} t \rightarrow-\infty$ when $Q_{\mathrm{b}}<-\Delta \rho / 8$ in the limit $b_{0} \rightarrow 0^{+}$.

\section{Appendix A: Expressions for the pressure gradients and the velocities of both fluids}

In this Appendix we obtain the expressions for the pressure gradients and velocities of both fluids.

With the pressures of both fluids, $p_{i}$ for $i=1,2$, satisfying (35), integration of the two equations in (9) twice with respect to $r$ introduces four unknown functions of $z$ and $t$, which, along with the two unknown pressure gradients, $\partial p_{i} / \partial z$, means that there are a total of six unknown functions to determine. These functions are determined from (16) on $r=0,(19)$ on $r=1,(22)$ and (26) with $T_{i}$ for $i=1,2$ given by (33) on $r=b$, and (30) with $Q_{i}$ for $i=1,2$ given by (6), together with $\partial p_{1} / \partial z-\partial p_{2} / \partial z=M z / b$ (obtained by differentiation of (36) with respect to $z$ ), leading to the expressions for the axial components of velocity $w_{i}$ and the pressure gradients $\partial p_{i} / \partial z$. Then integration of (7) subject to (15) on $r=0$ and (18) on $r=1$ leads to expressions for the radial components of velocity $u_{i}$ (which 
automatically satisfy $(21)$ on $r=b)$. Specifically, the pressure gradients are given by

$$
\frac{\partial p_{1}}{\partial z}=\frac{M m z\left(1-b^{2}\right)\left(1+3 b^{2}\right)-\Delta \rho m b\left(1-b^{2}\right)^{2}-8 Q_{\mathrm{b}} m b}{b\left[m-(m-1) b^{4}\right]}-\rho_{1}, \quad \frac{\partial p_{2}}{\partial z}=\frac{\partial p_{1}}{\partial z}-\frac{M z}{b},
$$

and the velocity components are given by

$$
\begin{gathered}
u_{1}=\frac{M r}{16 b}\left[-\frac{\left(1-b^{2}\right)\left[4(m-1) b^{4}+b^{2}\left(3 r^{2}-4 m-2\right)+r^{2}\right]}{m-(m-1) b^{4}}+4 b^{2} \ln b\right] \\
w_{1}=\frac{M z}{4 b}\left[\frac{\left(1-b^{2}\right)\left[2(m-1) b^{4}+b^{2}\left(3 r^{2}-2 m-1\right)+r^{2}\right]}{m-(m-1) b^{4}}-2 b^{2} \ln b\right] \\
+\frac{\Delta \rho}{4}\left[\frac{\left(1-b^{2}\right)\left[2(m-1) b^{4}+b^{2}\left(r^{2}-2 m+1\right)-r^{2}\right]}{m-(m-1) b^{4}}-2 b^{2} \ln b\right]+\frac{2 Q_{\mathrm{b}}\left[(1-m) b^{2}+m-r^{2}\right]}{m-(m-1) b^{4}}, \\
u_{2}=\frac{M b}{16 r}\left[-\frac{\left(1-r^{2}\right)\left[2(m-1) b^{4}-(2 m+1) b^{2}\left(1-r^{2}\right)-2 m r^{2}\right]}{m-(m-1) b^{4}}+4 r^{2} \ln r\right] \\
+\frac{\Delta \rho b^{2}}{4}\left[\frac{\left(1-r^{2}\right)\left[(2 m-1) b^{2}-2 m\right]}{m-(m-1) b^{4}}-2 \ln r\right]+\frac{2 Q_{\mathrm{b}} m\left(1-r^{2}\right)}{m-(m-1) b^{4}} .
\end{gathered}
$$

Figure 11 shows the radial velocity $u_{1}$ and the normalised axial velocity $w_{1} / z$ at the interface $r=b$ when only Marangoni effects are present plotted as functions of $b$ for the full range of values of $m$ in the case $M=1, Q_{\mathrm{b}}=0$ and $\Delta \rho=0$. In particular, Figure 11 shows that $u_{1}$ and $w_{1}$ are non-monotonic functions of $b$ satisfying $u_{1}<0$ for $0<b<b_{\mathrm{c}}, u_{1}>0$ for $b_{\mathrm{c}}<b<1$, and $w_{1}>0$ for $0<b<1$.

\section{Appendix B: Corresponding results for a two-dimensional droplet in a channel}

In this Appendix for completeness we briefly summarise the corresponding results for the closely related problem of a two-dimensional droplet with constant volume (per unit width) $V=2 b L$, semi-width $b=b(t)$ and length $L=L(t)$ in a non-uniformly heated channel of constant semi-width $a$.

Referred to Cartesian coordinates $O x y z$, with the $z$-axis vertically upwards along the centreline of the channel, the walls of the channel are at $x= \pm a$, and, since problem is symmetric about $x=0$, we need only consider the flow in $0 \leq x \leq a$. The geometry of the problem is as sketched in Figure 2, but with the coordinate $r$ replaced by $x$. The characteristic axial length scale $\ell$ and the appropriate non-dimensionalisation are given by (4) and (5), respectively, with $r$ replaced by $x$ and $\pi a^{2}$ replaced by $2 a$.

The leading-order governing equations corresponding to (7)-(10) are

$$
\frac{\partial u_{1}^{*}}{\partial x^{*}}+\frac{\partial w_{1}^{*}}{\partial z^{*}}=0, \quad \frac{\partial u_{2}^{*}}{\partial x^{*}}+\frac{\partial w_{2}^{*}}{\partial z^{*}}=0
$$




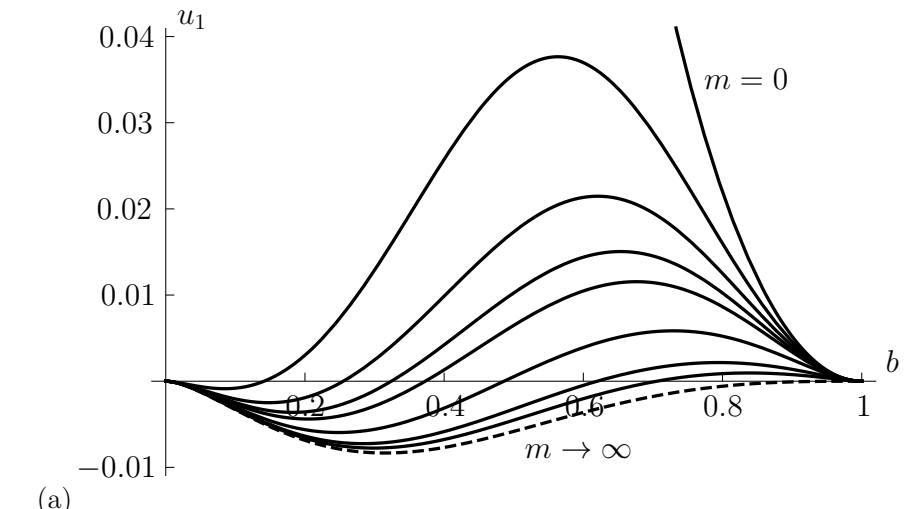

(a)

(b)

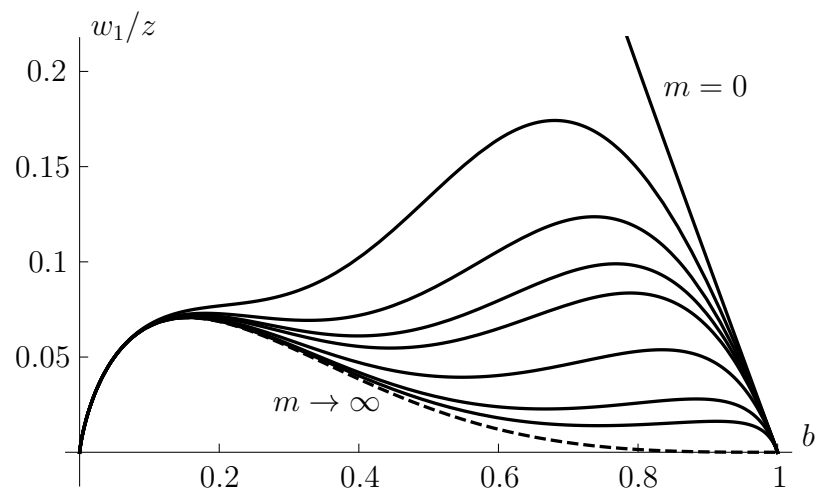

FIG. 11. Plots of (a) the radial velocity $u_{1}$ and (b) the normalised axial velocity $w_{1} / z$ at the interface $r=b$ given by (A2) and (A3), respectively, when only Marangoni effects are present as functions of $b$ for $m=0,1 / 4,1 / 2,3 / 4,1,2,5$ and 10 and in the limit $m \rightarrow \infty$ (the latter shown dashed) in the case $M=1, Q_{\mathrm{b}}=0$ and $\Delta \rho=0$.

$$
\begin{array}{cc}
\frac{\partial p_{1}^{*}}{\partial x^{*}}=0, & \frac{\partial p_{2}^{*}}{\partial x^{*}}=0 \\
m \frac{\partial^{2} w_{1}^{*}}{\partial x^{* 2}}=\frac{\partial p_{1}^{*}}{\partial z^{*}}+\rho_{1}^{*}, & \frac{\partial^{2} w_{2}^{*}}{\partial x^{* 2}}=\frac{\partial p_{2}^{*}}{\partial z^{*}}+\rho_{2}^{*}, \\
\frac{\partial^{2} T_{1}^{*}}{\partial x^{* 2}}=0, & \frac{\partial^{2} T_{2}^{*}}{\partial x^{* 2}}=0
\end{array}
$$

and are subject to the leading-order boundary conditions (15)-(26) with $r^{*}$ replaced by $x^{*}$ where appropriate, except for (25), which is replaced by simply

$$
p_{1}^{*}-p_{2}^{*}=0
$$

The semi-width of the droplet, $b^{*}$, now satisfies the kinematic condition

$$
\frac{\mathrm{d} b^{*}}{\mathrm{~d} t^{*}}+\frac{\partial Q_{1}^{*}}{\partial z^{*}}=0, \quad \text { or, equivalently, } \quad \frac{\mathrm{d} b^{*}}{\mathrm{~d} t^{*}}-\frac{\partial Q_{2}^{*}}{\partial z^{*}}=0
$$

while the volume of the droplet is now given by $b^{*} L^{*}=1$, and hence (32) is replaced by

$$
L^{*}=\frac{1}{b^{*}}
$$


Dropping the stars on dimensionless quantities, the temperatures of both fluids are again given by (33), the pressure gradients are given by

$$
\frac{\partial p_{1}}{\partial z}=\frac{\partial p_{2}}{\partial z}=\frac{3 M m z\left(1-b^{2}\right)-\Delta \rho m(1-b)^{2}(2+b)-3 Q_{\mathrm{b}} m}{2\left[m-(m-1) b^{3}\right]}-\rho_{1},
$$

and the velocity components are given by

$$
\begin{gathered}
u_{1}=\frac{M(1-b)\left[3 b-1-(m-1)(1-b)^{3}-(1+b) x^{2}\right] x}{4\left[m-(m-1) b^{3}\right]}, \\
w_{1}=\frac{M z(1-b)\left[4 b^{3}+m(1-b)^{3}\right]+\Delta \rho b(1-b)^{2}\left[m(1-b)^{2}-2 b^{2}\right]+3 Q_{\mathrm{b}} m\left(1-b^{2}\right)}{4\left[m-(m-1) b^{3}\right]} \\
-\frac{\left[3 M z\left(1-b^{2}\right)+\Delta \rho(1-b)^{2}(2+b)+3 Q_{\mathrm{b}}\right]\left(b^{2}-x^{2}\right)}{4\left[m-(m-1) b^{3}\right]}, \\
u_{2}=-\frac{M\left[m\left(1-b^{2}\right) x-2 b^{2}(m-(m-1) b)\right](1-x)^{2}}{4\left[m-(m-1) b^{3}\right]}, \\
w_{2}=(M z+\Delta \rho b)(1-x)-\frac{\left[3 M m z\left(1-b^{2}\right)+\Delta \rho b\left(3 m\left(1-b^{2}\right)+2 b^{2}\right)-3 Q_{\mathrm{b}} m\right]\left(1-x^{2}\right)}{4\left(m-(m-1) b^{3}\right)} .
\end{gathered}
$$

Figure 12 shows the transverse velocity $u_{1}$ and the normalised axial velocity $w_{1} / z$ at the interface $r=b$ when only Marangoni effects are present plotted as functions of $b$ for in the case $M=1, Q_{\mathrm{b}}=0$ and $\Delta \rho=0$. In particular, Figure 12 shows that $u_{1}$ and $w_{1}$ are non-monotonic functions of $b$ satisfying $u_{1}<0$ for $0<b<b_{\mathrm{c}}, u_{1}>0$ for $b_{\mathrm{c}}<b<1, w_{1}>0$ for $0<b<1$, and $w_{1} \rightarrow(M / 4)^{-}$as $b \rightarrow 0^{+}$(the latter of which is, slightly unexpectedly, qualitatively different from the corresponding behaviour in the axisymmetric case shown in Figure 11).

The axial volume fluxes of fluid 1 and fluid 2, respectively, are given by

$$
Q_{1}=2 \int_{0}^{b} u_{1} \mathrm{~d} x, \quad Q_{2}=2 \int_{b}^{1} u_{2} \mathrm{~d} x
$$

which lead again to (37), but with the functions $f_{j}$ for $j=\mathrm{M}, \mathrm{G}$, B now defined by

$$
\begin{gathered}
f_{\mathrm{M}}(b, m)=\frac{(1-b)^{2} b\left[m(1-b)^{2}-2 b^{2}\right]}{2\left[m-(m-1) b^{3}\right]}, \\
f_{\mathrm{G}}(b, m)=\frac{(1-b)^{3} b^{2}[3 m(1-b)+4 b]}{6\left[m-(m-1) b^{3}\right]}, \\
f_{\mathrm{B}}(b, m)=\frac{b\left[3 m\left(1-b^{2}\right)+2 b^{2}\right]}{2\left[m-(m-1) b^{3}\right]} .
\end{gathered}
$$

These $f_{j}$ are qualitatively similar to the corresponding functions in the axisymmetric case given by (38)-(40) and shown in Figure 3.

The evolution of the semi-width of the droplet, $b(t)$, is governed by the equation

$$
\frac{\mathrm{d} b}{\mathrm{~d} t}=-M f_{\mathrm{M}}(b, m)
$$




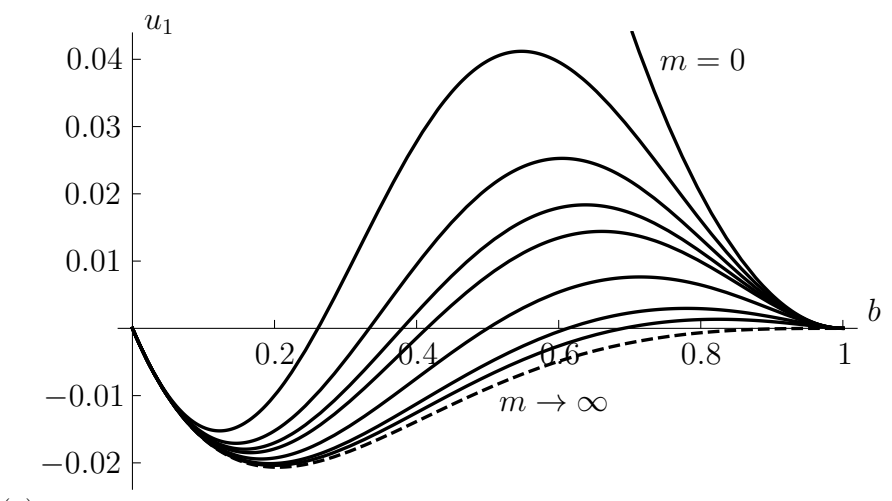

(a)

(b)

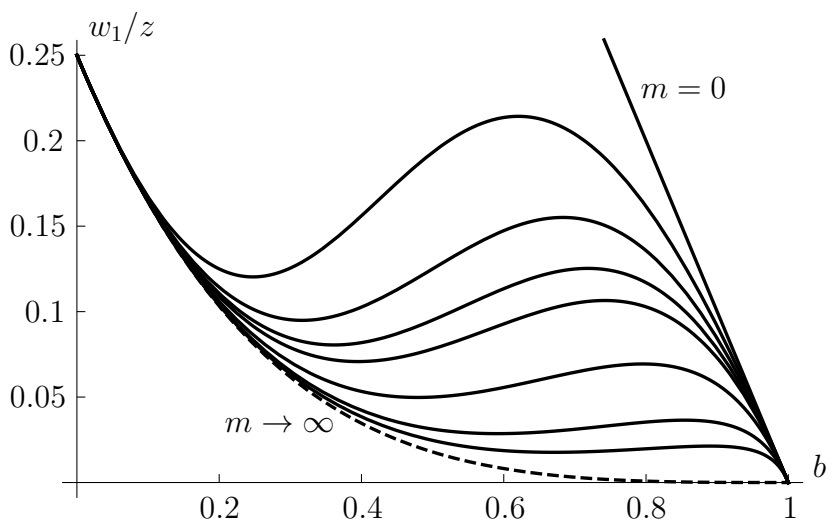

FIG. 12. Plots of (a) the transverse velocity $u_{1}$ and (b) the normalised axial velocity $w_{1} / z$ at the interface $x=b$ given by (B9) and (B10), respectively, when only Marangoni effects are present as functions of $b$ for $m=0,1 / 4,1 / 2,3 / 4,1,2,5$ and 10 and in the limit $m \rightarrow \infty$ (the latter shown dashed) in the case $M=1, Q_{\mathrm{b}}=0$ and $\Delta \rho=0$.

which has the implicit solution $t=t(b)$ given by

$$
M t=\phi(b)-\phi\left(b_{0}\right)
$$

where the function $\phi(b)$ is defined by

$$
\phi=\frac{1}{1-b}+\frac{3 m+2}{2} \ln \left(m-\frac{2 b^{2}}{(1-b)^{2}}\right)+\frac{7 \sqrt{m}}{2 \sqrt{2}} \ln \frac{b-b_{\mathrm{c}}}{\hat{b}_{\mathrm{c}}-b}-2 \ln \frac{b}{1-b},
$$

in which $b_{\mathrm{c}}=b_{\mathrm{c}}(m)$ and $\hat{b}_{\mathrm{c}}=\hat{b}_{\mathrm{c}}(m)$ are the roots of $f_{\mathrm{M}}(b, m)=0$ given by

$$
b_{\mathrm{c}}=\frac{\sqrt{m}}{\sqrt{m}+\sqrt{2}}, \quad \hat{b}_{\mathrm{c}}=\frac{\sqrt{m}}{\sqrt{m}-\sqrt{2}} .
$$

The evolution of $b$, and hence of $L$, given by (B18) is qualitatively similar to that in the axisymmetric case shown in Figure 5.

The evolution of the position of the centre of mass of the droplet, $c(t)$, is governed by

$$
\frac{\mathrm{d} c}{\mathrm{~d} t}=\frac{M c f_{\mathrm{M}}+\Delta \rho f_{\mathrm{G}}+Q_{\mathrm{b}} f_{\mathrm{B}}}{b}
$$

which has a parametric solution for $c$ (with parameter $b$ ) given by

$$
c=\frac{b_{0} c_{0}}{b}+\frac{1}{b}\left(\psi(b)-\psi\left(b_{0}\right)\right)
$$


where the function $\psi(b)$ is defined by

$$
\begin{gathered}
\psi=\frac{1}{M}\left\{\frac{Q_{\mathrm{b}}}{1-b}+\frac{(1-b)\left[3 m^{2}(1+b)-2 m(5 b-3)-8(1-b)\right] \Delta \rho}{6(m-2)^{2}}-3 m Q_{\mathrm{b}} \ln (1-b)\right. \\
\left.-\sqrt{2 m}\left[Q_{\mathrm{b}}+\frac{\left(3 m^{2}+6 m-8\right) \Delta \rho}{6(m-2)^{3}}\right] \ln \frac{\hat{b}_{\mathrm{c}}-b}{b-b_{\mathrm{c}}}+m\left[\frac{3 Q_{\mathrm{b}}}{2}-\frac{(7 m-6) \Delta \rho}{3(m-2)^{3}}\right] \ln \left[\left(\hat{b}_{\mathrm{c}}-b\right)\left(b-b_{\mathrm{c}}\right)\right]\right\} .
\end{gathered}
$$

The evolution of $c(t)$, and hence of $z_{\mathrm{f}}(t)$ and $z_{\mathrm{b}}(t)$, given by (B22) is qualitatively similar to that in the axisymmetric case shown in Figures 6, 7, 9 and 10. 